Algebraic $8 \mathcal{G}$ Geometric $\mathcal{T}_{\text {opology }}$

Volume 5 (2005) 301-354

Published: 17 April 2005

ATG

\title{
The periodic Floer homology of a Dehn twist
}

\author{
Michael Hutchings \\ Michael Sullivan
}

\begin{abstract}
The periodic Floer homology of a surface symplectomorphism, defined by the first author and M. Thaddeus, is the homology of a chain complex which is generated by certain unions of periodic orbits, and whose differential counts certain embedded pseudoholomorphic curves in $\mathbb{R}$ cross the mapping torus. It is conjectured to recover the Seiberg-Witten Floer homology of the mapping torus for most spin-c structures, and is related to a variant of contact homology. In this paper we compute the periodic Floer homology of some Dehn twists.
\end{abstract}

AMS Classification 57R58; 53D40, 57R50

Keywords Periodic Floer homology, Dehn twist, surface symplectomorphism

\section{Introduction}

Let $\Sigma$ be a compact surface, possibly with boundary, with a symplectic form $\omega$, and let $\phi$ be a symplectomorphism of $\Sigma$. Consider the mapping torus

$$
Y_{\phi}:=\frac{[0,1] \times \Sigma}{(1, x) \sim(0, \phi(x))} .
$$

Projection onto the $[0,1]$ factor defines a fibration $Y_{\phi} \rightarrow \mathbb{R} / \mathbb{Z}$ with fiber $\Sigma$. There is a natural flow $R$ on $Y_{\phi}$ which increases the $[0,1]$ coordinate, and we identify periodic orbits of $\phi$ with closed orbits of this flow in $Y_{\phi}$. We fix a homology class $h \in H_{1}\left(Y_{\phi}\right)$, and define the degree $d$ to be the intersection number of $h$ with a fiber of $Y_{\phi} \rightarrow \mathbb{R} / \mathbb{Z}$. Under "monotonicity" and " $d$-regularity" assumptions on $\phi$, we can define the periodic Floer homology $(\mathrm{PFH})$, denoted by $H P_{*}(\phi, h)$; see [12, the background in [9], and the review in Section 2, Roughly speaking, this is the homology of a chain complex which is generated by certain unions of periodic orbits of $\phi$ with total homology class $h$, and whose differential counts certain embedded pseudoholomorphic curves in $\mathbb{R} \times Y_{\phi}$ for a suitable almost complex structure $J$. It is expected that the PFH is independent of $J$ and invariant under appropriate isotopy of $\phi$ fixing $\left.\phi\right|_{\partial \Sigma}$. 
When $d=1$, PFH reverts to the ordinary symplectic Floer homology of $\phi$, which is the homology of a chain complex generated by fixed points of $\phi$ and whose differential counts pseudoholomorphic sections of $\mathbb{R} \times Y_{\phi} \rightarrow \mathbb{R} \times S^{1}$. The symplectic Floer homology has been computed for a Dehn twist by Seidel [21, more examples have been computed by Eftekhary [4] and Gautschi [8], and a conjecture for a product of positive Dehn twists on a surface with boundary is given in 22$]$.

Results In this paper we compute the periodic Floer homology of some Dehn twists. We begin, in Theorem 3.1, by computing the PFH of a perturbation of the "positive" twist on the cylinder

$$
\begin{aligned}
\phi_{0}:\left[X_{1}, X_{2}\right] \times S^{1} & \longrightarrow\left[X_{1}, X_{2}\right] \times S^{1}, \\
(x, y) & \longmapsto(x, y-x)
\end{aligned}
$$

for any $J$. Here, as elsewhere in this paper, we identify $S^{1}=\mathbb{R} / \mathbb{Z}$. In fact, in Theorem 3.5] we obtain a combinatorial formula for most of the differential in the chain complex, in terms of "rounding corners" of convex polygonal paths connecting lattice points in the plane. In the terminology of Section 2, this part of the differential counts genus zero pseudoholomorphic curves with two "incoming ends", any number of "outgoing ends", possibly together with "trivial cylinders". We prove these theorems in Section 3 .

In Theorem 4.1 proved in Section 4, we compute the PFH of an $n$-fold positive Dehn twist on the torus (for any $J$ ), namely a perturbation of

$$
\begin{aligned}
\phi_{0}^{T}:(\mathbb{R} / n \mathbb{Z}) \times(\mathbb{R} / \mathbb{Z}) & \longrightarrow(\mathbb{R} / n \mathbb{Z}) \times(\mathbb{R} / \mathbb{Z}), \\
(x, y) & \longmapsto(x, y-x) .
\end{aligned}
$$

In Section [5 we consider a composition $\phi^{\Sigma}$ of Dehn twists along disjoint circles $\gamma_{i}$ on a higher genus (connected) surface $\Sigma$, perturbed so that it is close to the identity away from the circles $\gamma_{i}$. To ensure monotonicity of the standard representative of the composition of Dehn twists, we assume:

$(*)$ If $\xi \in H_{1}(\Sigma)$ has nonzero intersection number with some $\left[\gamma_{i}\right]$, then $\phi_{*}^{\Sigma} \xi \neq$ $\xi$ in $H_{1}(\Sigma)$. Also, $d \neq g(\Sigma)-1$ when $\partial \Sigma=\emptyset$.

If we further assume that

(**) each component of $\Sigma \backslash \bigcup_{i} \gamma_{i}$ contains a component of $\partial \Sigma$ or has sufficiently large genus with respect to $d$,

then the $\mathrm{PFH}$ can be computed in terms of the cylinder complex described above and Morse theory on $\Sigma \backslash \bigcup_{i} \gamma_{i}$. This is complicated in general, but we work out the cases when $\Sigma$ is closed and there is one circle $\gamma_{i}$, nonseparating or separating, in Theorems 5.3 and 5.4 respectively. 
Motivation There are three basic motivations for this paper. First, it is conjectured in [12] that if $\Sigma$ is closed and connected and $d<g(\Sigma)-1$, then $H P_{*}(\phi, h)$ is isomorphic to the Seiberg-Witten Floer (SWF) homology of $Y_{\phi}$ for a spin-c structure corresponding to $h$. This conjecture is an analogue of Taubes's "SW=Gr" theorem [24, relating Seiberg-Witten solutions to embedded pseudoholomorphic curves on closed symplectic 4-manifolds, for the noncompact symplectic 4-manifold $\mathbb{R} \times Y_{\phi}$. This conjecture is known to hold at the level of Euler characteristics as a consequence of Taubes's theorem applied to $S^{1} \times Y_{\phi}$, see [10]. It also fits nicely with a conjecture of Salamon [19] relating SWF homology of mapping tori to symplectic Floer homology of induced maps on symmetric products. Thus our results conjecturally give some new examples of SWF homology. The version of SWF homology considered here is conjectured to agree with the invariant $\mathrm{HF}^{+}$of Ozsváth-Szabó [16, 17].

Second, one should be able to define a "quantum product" relating the $\mathrm{PFH}$ of three surface diffeomorphisms $f, g$, and $f \circ g$, cf. [3], and then in principle recover the Seiberg-Witten invariants of a 4-dimensional symplectic Lefschetz pencil by taking the quantum products of certain elements in the PFH of a Dehn twist. Thus the PFH of a Dehn twist appears to be a fundamental building block in invariants of symplectic 4-manifolds.

Third, one can define an analogue of PFH for a contact 3-manifold, thus obtaining an interesting variant of the symplectic field theory of [5], which we call "embedded contact homology". Some basic contact 3-manifolds, such as $S^{1} \times S^{2}$ with the overtwisted contact structure considered in [25], or the unit cotangent bundle of $T^{2}$, contain pieces whose Reeb flow is diffeomorphic to the mapping torus flow $R$ for the inverse of the cylinder twist (2). Thus we expect the techniques developed in this paper to be useful in computing more examples of various flavors of contact homology.

Remarks on the proofs We organize the computations in this paper using some spectral sequences, most of which are variations on the following theme. Suppose that our symplectomorphism $\phi$ has an invariant curve $\xi \subset \Sigma$, on which $\phi$ is an irrational rotation, and which divides $\Sigma$ into pieces $\Sigma_{1}$ and $\Sigma_{2}$. Then in some cases, there is a spectral sequence $\mathcal{E}_{*, *}^{*}$ which converges to $H P_{*}(\phi, h)$, with

$$
\mathcal{E}^{1} \simeq \bigoplus_{h_{1}+h_{2}=h} H P_{*}\left(\left.\phi\right|_{\Sigma_{1}}, h_{1}\right) \otimes H P_{*}\left(\left.\phi\right|_{\Sigma_{2}}, h_{2}\right)
$$

One such spectral sequence is used in Section 3.9 to compute the PFH of a twist on a cylinder, by studying how the PFH changes as one twists the symplectomorphism on the boundary. Other such spectral sequences are used for 
the torus in Section 4.1 and for higher genus surfaces in Section 5.3 and Section 5.4 to cut out the cylinders in which twisting takes place.

In some cases a spectral sequence satisfying (4) exists but might not converge to $H P_{*}(\phi, h)$, roughly speaking due to higher genus pseudoholomorphic curves which "wrap around" large parts of $\Sigma$. This failure to converge is measured by the "wrapping spectral sequence" introduced in Section 4.1. We make the hypothesis $(* *)$ in order to avoid dealing with these higher genus curves by ensuring that the wrapping spectral sequence degenerates.

An simple but important trick used in several places is the "local energy inequality" of Section 3.4. inspired by Gautschi [8, which gives homological constraints on slices of pseudoholomorphic curves, roughly from the fact that neighborhoods of these slices have positive energy.

In this paper we use a number of general results about pseudohomolomorphic curves in $\mathbb{R} \times Y$ which are proved in [9], so it may be helpful to have a copy of the latter paper on hand.

Update Since the first version of this paper was distributed, the following related developments have occurred.

In [1], we used the techniques of this paper to calculate the embedded contact homology of $T^{3}$ in terms of more general combinatorial chain complexes involving rounding corners of polygons. Also, [1] explains how to do such calculations over $\mathbb{Z}$, instead of over $\mathbb{Z} / 2$ as in the present paper. It follows easily from [1] that all homology calculations in the present paper hold over $\mathbb{Z}$, with all $\mathbb{Z} / 2$ summands replaced by $\mathbb{Z}$.

Jabuka and Mark [13, using results of Ozsváth and Szabó [18, computed the Ozsváth-Szabó Floer homology $\mathrm{HF}^{+}$of the mapping tori of some Dehn twists and compositions thereof on closed surfaces $\Sigma$. If $d<g(\Sigma)-1$, then the PFH agrees with $\mathrm{HF}^{+}$of the mapping torus in those cases where both have been calculated. This provides some nontrivial evidence for the conjectured relation between PFH and Seiberg-Witten Floer homology.

Acknowledgments We thank D. Canary, Y. Eliashberg, R. Gautschi, H. Hofer, A. Ivrii, L. Mosher and M. Skandera for helpful discussions.

The first author was partially supported by NSF grant DMS-0204681 and the Alfred P. Sloan Foundation. The second author was partially supported by NSF grant DMS-0305825 and a VIGRE fellowship. 


\section{Review of periodic Floer homology}

Let $\Sigma$ be a compact surface, possibly with boundary, and let $\omega$ be a symplectic form on $\Sigma$. Let $\phi$ be a symplectomorphism of $(\Sigma, \omega)$. Let $Y=Y_{\phi}$ denote the mapping torus of $\phi$ as in (11), and pick a "sector" $h \in H_{1}(Y)$. Under the "monotonicity" and "regularity" assumptions below, we now define the periodic Floer homology $H P_{*}(\phi, h)$. This is the homology of a chain complex which is generated by "admissible orbit sets" and graded by the relative index $I$, and whose differential counts "flow lines", all defined below.

We use the following notation. Let $V \rightarrow Y$ denote the vertical tangent bundle of $Y \rightarrow S^{1}$. Let $[\Sigma] \in H_{2}(Y)$ denote the homology class of a fiber, and define the degree $d:=h \cdot[\Sigma] \in \mathbb{Z}$. Define the "index ambiguity class"

$$
c(h):=c_{1}(V)+2 h^{\times} \in H^{2}(Y ; \mathbb{Z}),
$$

where $h^{\times}$denotes the image of $h$ under $H_{1}(Y) \rightarrow H_{1}(Y, \partial Y) \simeq H^{2}(Y ; \mathbb{Z})$. Let $R$ denote the flow on $Y$ that increases the $[0,1]$ coordinate in (11).

Orbit sets An orbit set is a finite set of pairs $\left\{\left(\alpha_{i}, m_{i}\right)\right\}$ where the $\alpha_{i}$ 's are distinct (nondegenerate) irreducible periodic orbits of $\phi$, regarded as embedded oriented circles in $Y$ tangent to $R$, and the $m_{i}$ 's are positive integers, which can be thought of as "multiplicities". The orbit set $\left\{\left(\alpha_{i}, m_{i}\right)\right\}$ is admissible if $m_{i}=1$ whenever $\alpha_{i}$ is hyperbolic, i.e. the linearized return map of $\phi$ along $\alpha_{i}$ has real eigenvalues. We define the homology class $[\alpha]:=\sum_{i} m_{i}\left[\alpha_{i}\right] \in H_{1}(Y)$, and we let $\mathcal{A}(h)$ denote the set of admissible orbit sets $\alpha$ with $[\alpha]=h$. We often denote an orbit set by a commutative product $\alpha_{1}^{m_{1}} \cdots \alpha_{k}^{m_{k}}$, although the index and differential defined below are not well-behaved with respect to this sort of multiplication.

The relative index Let $\alpha=\left\{\left(\alpha_{i}, m_{i}\right)\right\}$ and $\beta=\left\{\left(\beta_{j}, n_{j}\right)\right\}$ be orbit sets with $[\alpha]=[\beta]$. Let $H_{2}(Y ; \alpha, \beta)$ denote the set of relative homology classes of 2-chains $W$ in $Y$ with

$$
\partial W=\sum_{i} m_{i} \alpha_{i}-\sum_{j} n_{j} \beta_{j}
$$

this is an affine space over $\mathrm{H}_{2}(Y)$. If $Z \in \mathrm{H}_{2}(Y ; \alpha, \beta)$, define the relative index

$$
I(\alpha, \beta ; Z):=c_{\tau}(Z)+Q_{\tau}(Z)+\mu_{\tau}(\alpha, \beta) \in \mathbb{Z} .
$$


Here $\tau$ is a homotopy class of symplectic trivialization of $V$ over the $\alpha_{i}$ 's and $\beta_{j}$ 's; $c_{\tau}(Z)$ denotes the relative first Chern class of the bundle $V$ over the relative homology class $Z$ with respect to the boundary trivialization $\tau$; $Q_{\tau}(Z)=Q_{\tau}(Z, Z)$ denotes the relative intersection pairing in $[0,1] \times Y$; and

$$
\mu_{\tau}(\alpha, \beta):=\sum_{i} \sum_{k=1}^{m_{i}} \mu_{\tau}\left(\alpha_{i}^{k}\right)-\sum_{j} \sum_{k=1}^{n_{j}} \mu_{\tau}\left(\beta_{j}^{k}\right),
$$

where $\mu_{\tau}\left(\gamma^{k}\right)$ is the Conley-Zehnder index of the $k^{\text {th }}$ iterate of $\gamma$. These notions are explained in detail in [9, Section 2].

The relative index has the following basic properties [9, Prop. 1.6]. First, the definition does not depend on $\tau$. Second, if $\gamma$ is another homologous orbit set and $W \in H_{2}(Y ; \beta, \gamma)$, then we have the additivity property

$$
I(\alpha, \beta ; Z)+I(\beta, \gamma ; W)=I(\alpha, \gamma ; Z+W) .
$$

Third, if $\alpha$ and $\beta$ are admissible, then

$$
I(\alpha, \beta ; Z) \equiv \sum_{i} \epsilon\left(\alpha_{i}\right)-\sum_{j} \epsilon\left(\beta_{j}\right) \quad \bmod 2,
$$

where $(-1)^{\epsilon}$ denotes the Lefschetz sign, which is -1 for hyperbolic orbits with positive eigenvalues and +1 otherwise. Fourth, we have the "index ambiguity formula"

$$
I(\alpha, \beta ; Z)-I(\alpha, \beta ; W)=\langle Z-W, c(h)\rangle .
$$

Monotonicity The symplectic form $\omega$ on $\Sigma$ induces a symplectic structure on $V$ which canonically extends to a closed 2-form on $Y$, which we still denote by $\omega$, with $R-^{\prime} \omega=0$. We say that $(\phi, h)$ is monotone if

$$
[\omega]=\lambda c(h) \in H^{2}(Y ; \mathbb{R}),
$$

where $\lambda \in \mathbb{R}$. An elementary calculation in [12] shows that for any given $h$, if $\Sigma$ is connected and $\partial \Sigma \neq \emptyset$ or $d \neq g(\Sigma)-1$, then one can achieve monotonicity by a symplectic isotopy of $\phi$ fixing $\left.\phi\right|_{\partial \Sigma}$.

Almost complex structure An almost complex structure $J$ on $\mathbb{R} \times Y$ is admissible if:

- $J\left(\partial_{s}\right)=R$, where $s$ denotes the $\mathbb{R}$ coordinate.

- $J$ is invariant under the obvious $\mathbb{R}$-action on $\mathbb{R} \times Y$.

- $J$ is tamed by $\Omega:=\omega+d s \wedge d t$, that is $\Omega(v, J v)>0$ for $v \neq 0$. 
We say that $(\phi, J)$ is $d$-regular if $J$ is admissible and:

- Each periodic orbit of period $p \leq d$ is nondegenerate.

- (Local linearity) For each periodic orbit of period $p \leq d$, there are local coordinates on $\Sigma$ near $\gamma$ for which $\phi^{p}$ is linear; and there is a tubular neighborhood $N$ of $\gamma$ on which $J$ sends $V$ to $V$, by a constant matrix on each fiber of the projection $N \rightarrow \gamma$ induced by the projection $Y \rightarrow S^{1}$. If $\gamma$ is elliptic, then $J$ is invariant under the flow $R$ in $N$.

- Near each component of $\partial \Sigma$ there are local coordinates $x \in(-\epsilon, 0], y \in$ $\mathbb{R} / \mathbb{Z}$ with $J\left(\partial_{x}\right)=\partial_{y}$ and $\phi(x, y)=(x, y+\theta)$, where $q \theta \notin \mathbb{Z}$ for all integers $1 \leq q \leq d$.

- $J$ is sufficiently close to sending $V$ to $V$.

The local linearity condition might not be necessary but is used in [9, 12, to simplify the analysis. The next condition ensures that flow lines (defined below) do not approach the boundary, by the maximum principle. The last condition is used in [9] to rule out bubbling of closed pseudoholomorphic curves in moduli spaces of flow lines.

Flow lines We now consider $J$-holomorphic curves in $\mathbb{R} \times Y$, where $J$ is admissible. The simplest example of a $J$-holomorphic curve in $\mathbb{R} \times Y$ is $\mathbb{R} \times \gamma$, where $\gamma$ is a periodic orbit; we call this a trivial cylinder. More generally, if $C$ is a $J$-holomorphic curve, an outgoing end at $\gamma$ of multiplicity $m$ is an end of $C$ asymptotic to $\mathbb{R}^{+} \times \gamma^{m}$ as $s \rightarrow+\infty$, where $\gamma^{m}$ denotes a connected $m$-fold cover of $\gamma$. Incoming ends are defined analogously with $s \rightarrow-\infty$.

Let $\alpha=\left\{\left(\alpha_{i}, m_{i}\right)\right\}$ and $\beta=\left\{\left(\beta_{j}, n_{j}\right)\right\}$ be orbit sets with $[\alpha]=[\beta]$. A flow line from $\alpha$ to $\beta$ is a $J$-holomorphic curve $C \subset \mathbb{R} \times Y$ such that:

- $C$ is embedded, except that trivial cylinders may be repeated, although these are not allowed to intersect the rest of $C$.

- $C$ is a punctured compact Riemann surface and has outgoing ends at $\alpha_{i}$ of multiplicity $q_{i, k}$ with $\sum_{k} q_{i, k}=m_{i}$, incoming ends at $\beta_{j}$ of multiplicity $q_{j, k}^{\prime}$ with $\sum_{k} q_{j, k}^{\prime}=n_{j}$, and no other ends.

Let $\mathcal{M}(\alpha, \beta ; Z)$ denote the moduli space of flow lines from $\alpha$ to $\beta$ in the relative homology class $Z$, and $\mathcal{M}(\alpha, \beta):=\bigcup_{Z \in H_{2}(Y ; \alpha, \beta)} \mathcal{M}(\alpha, \beta ; Z)$. Note that $\mathbb{R}$ acts on these moduli spaces by translation in $\mathbb{R} \times Y$. If $C \in \mathcal{M}(\alpha, \beta)$, let $\mathcal{M}_{C}$ denote the component of the moduli space containing $C$. 
If $C \in \mathcal{M}(\alpha, \beta)$, we define $I(C):=I([C]):=I(\alpha, \beta ;[C])$. It is shown in 9 , Thm. 1.7], see also Proposition [2.1 below, that if $J$ is generic and $(\phi, J)$ is $d$-regular, where $d$ is the degree of $\alpha$ and $\beta$, then $\mathcal{M}_{C}$ is a manifold and

$$
\operatorname{dim}\left(\mathcal{M}_{C}\right) \leq I(C) .
$$

Moreover, equality holds only if $C$ is "admissible", see [9, Section 4]. If $C$ has no trivial cylinders, then "admissible" means that $\left\{q_{i, 1}, q_{i, 2}, \ldots\right\}=p_{\text {out }}\left(\alpha_{i}, m_{i}\right)$ and $\left\{q_{j, 1}^{\prime}, q_{j, 2}^{\prime}, \ldots\right\}=p_{\text {in }}\left(\beta_{j}, n_{j}\right)$, where to any periodic orbit $\gamma$ and positive integer $m$ there are a priori associated an "outgoing partition" $p_{\text {out }}(\gamma, m)$ and an "incoming partition" $p_{\text {in }}(\gamma, m)$ of $m$. A simple example which we will need later is that if $\gamma$ is elliptic, i.e. the linearized return map has eigenvalues $e^{ \pm 2 \pi i \theta}$, and if the linearized return map is a small (with respect to $d$ ) clockwise rotation, then for $m \leq d$,

$$
p_{\text {out }}(\gamma, m)=\{m\}, \quad p_{\text {in }}(\gamma, m)=\{1, \ldots, 1\} .
$$

So if $C$ is admissible and has no trivial cylinders, then $C$ can have at most one outgoing end at $\gamma$, while every incoming end of $C$ at $\gamma$ has multiplicity one.

The chain complex Assume that $(\phi, h)$ is monotone, and that $(\phi, J)$ is $d$ regular and $J$ is generic. We now define a chain complex $\left(C P_{*}(\phi, h), \delta\right)$, whose differential may depend on $J$. It is possible to define this over $\mathbb{Z}$, but for simplicity we will work over $\mathbb{Z} / 2$ in the present paper. The generators of the chain complex are admissible orbit sets:

$$
C P_{*}(\phi, h):=(\mathbb{Z} / 2)\{\mathcal{A}(h)\} .
$$

The differential is defined as follows: if $\alpha \in \mathcal{A}(h)$, then

$$
\delta \alpha:=\sum_{\beta \in \mathcal{A}(h)}\left(\sum_{I(\alpha, \beta ; Z)=1} \# \frac{\mathcal{M}(\alpha, \beta ; Z)}{\mathbb{R}}\right) \beta,
$$

where '\#' denotes the mod 2 count. By equations (7) and (8), all flow lines on the right hand side have the same integral of $\omega$, so by the compactness theorem of [9, Thm. 1.8], this is a finite sum. It will be shown in [12] that $\delta^{2}=0$. The homology of this chain complex is the periodic Floer homology $H P_{*}(\phi, h)$.

By equation (6), $H P_{*}(\phi, h)$ has a canonical $\mathbb{Z} / 2$ grading, which by (7) lifts noncanonically to a $\mathbb{Z} / N$ grading, where $N$ is the divisibility of $c(h)$ in $H^{2}(Y ; \mathbb{Z})$.

We expect that $H P_{*}(\phi, h)$ is independent of $J$ and invariant under symplectic isotopy of $\phi$ fixing $\left.\phi\right|_{\partial \Sigma}$ and preserving monotonicity. Moreover if $\Sigma$ is closed and connected and $d<g(\Sigma)-1$, then it is conjectured in [12 that $H P_{*}(\phi, h)$ agrees with the Seiberg-Witten Floer homology of $Y$ for a spin-c structure determined by $h$. 
Duality In this paper we mainly discuss positive Dehn twists, but one can easily deduce corresponding results for negative Dehn twists as follows. For any symplectomorphism $\phi$ of $\Sigma$, the self-diffeomorphism of $\mathbb{R} \times[0,1] \times \Sigma$ sending $(s, t, x) \mapsto(-s, 1-t, x)$ induces a symplectomorphism $\imath: \mathbb{R} \times Y_{\phi} \rightarrow \mathbb{R} \times Y_{\phi^{-1}}$, which sends a $d$-regular almost complex structure for $\phi$ to one for $\phi^{-1}$. Since incoming and outgoing ends of flow lines are switched, we obtain $H P_{*}\left(\phi^{-1}, \imath_{*} h\right) \simeq$ $H P^{-*}(\phi, h)$, where the right hand side is the "periodic Floer cohomology" defined using the dual differential.

Generalized flow lines In some technical arguments we need to consider generalized flow lines (GFL's), which are defined like flow lines except that we drop the embeddedness condition, see [9, Section 9.3]. We collect here the definitions and facts about GFL's that we will need.

If $C$ is a GFL, then in the notation from the paragraph on flow lines, we define

$$
\begin{aligned}
\mu_{\tau}^{0}(C) & :=\sum_{i} \sum_{k} \mu_{\tau}\left(\alpha_{i}^{q_{i, k}}\right)-\sum_{j} \sum_{k} \mu_{\tau}\left(\beta_{j}^{q_{j, k}^{\prime}}\right), \\
I^{\operatorname{vir}}(C) & :=-\chi(C)+2 c_{\tau}([C])+\mu_{\tau}^{0}(C) .
\end{aligned}
$$

If $C$ is a GFL from $\alpha$ to $\beta$, let $\operatorname{deg}(C)$ denote the degree of $\alpha$ and $\beta$.

We say that a GFL is a quasi-embedding if it is embedded except possibly at finitely many points. A connected GFL is either a quasi-embedding or a multiple cover. If $J$ is generic, then it follows from the index formula of [20] and a transversality argument in 9] that if $C$ is a quasi-embedding, then $I^{\mathrm{vir}}(C)$ equals the dimension of the component of the moduli space of quasi-embedded GFL's containing $C$. If $C$ is a flow line, and if $J$ is generic and $(\phi, J)$ is $\operatorname{deg}(C)$-regular, then

$$
\operatorname{dim}\left(\mathcal{M}_{C}\right) \leq I^{\operatorname{vir}}(C) .
$$

This is an inequality, because a moduli space of embedded flow lines can appear as a stratum in a moduli space of GFL's, see [9, Section 5].

If $C$ is a quasi-embedded GFL, then by [9, Section 3], we have the relative adjunction formula

$$
c_{\tau}([C])=\chi(C)+Q_{\tau}([C])+w_{\tau}(C)-2 \delta(C) .
$$

Here the integer $\delta(C)$ is a weighted count of the singularities of $C$, which satisfies $\delta(C) \geq 0$ with equality if and only if $C$ is embedded. Also, $w_{\tau}(C)$ is the signed sum of the writhes of the asymptotic braids of $C$, see [9. Section $3.1]$. 
It is shown in [9, Section 6] that if $C^{\prime}$ is a quasi-embedded GFL without trivial cylinders, and if $(\phi, J)$ is $\operatorname{deg}\left(C^{\prime}\right)$-regular, then

$$
w_{\tau}\left(C^{\prime}\right) \leq \mu_{\tau}(\alpha, \beta)-\mu_{\tau}^{0}\left(C^{\prime}\right) .
$$

Equations (5), (11), (13), and (14) imply that

$$
I^{\mathrm{vir}}\left(C^{\prime}\right) \leq I\left(C^{\prime}\right)-2 \delta\left(C^{\prime}\right) .
$$

It is also shown in [9, Section 7] that if $C^{\prime}$ is a quasi-embedded GFL without trivial cylinders and if $T$ is a union of trivial cylinders, then

$$
I\left(C^{\prime}\right) \leq I\left(C^{\prime} \cup T\right)-2 \#\left(C^{\prime} \cap T\right) .
$$

Here '\#' denotes the algebraic intersection number. Moreover, by intersection positivity [15,

$$
\#\left(C^{\prime} \cap T\right) \geq 0
$$

with equality if and only if $C^{\prime} \cap T=\emptyset$.

Observe that the inequalities (12), (15), and (16) imply the index inequality (9). The latter has the following generalization for multiply covered GFL's. This is proved in 9], but the proof below is much simpler.

Proposition 2.1 Let $C_{1}, \ldots, C_{k}$ be distinct, connected, quasi-embedded GFL's, and let $d_{1}, \ldots, d_{k}$ be positive integers. Suppose that $(\phi, J)$ is $d$-regular, where $d=\sum_{i} d_{i} \operatorname{deg}\left(C_{i}\right)$. Then

$$
\sum_{i=1}^{k} d_{i} I^{\mathrm{vir}}\left(C_{i}\right) \leq I\left(\sum_{i=1}^{k} d_{i}\left[C_{i}\right]\right)-2 \Delta,
$$

where

$$
\Delta=\sum_{i=1}^{k} d_{i}^{2} \delta\left(C_{i}\right)+\sum_{i<j} d_{i} d_{j} \#\left(C_{i} \cap C_{j}\right)
$$

Note that by intersection positivity, $\Delta \geq 0$, with equality if and only if the $C_{i}$ 's are embedded and disjoint.

Proof Suppose first that none of the $C_{i}$ 's is a trivial cylinder, so that $\mathbb{R}$ acts freely on the $C_{i}$ 's. Let $\widehat{C}_{i}$ be a union of $d_{i}$ distinct translates of $C_{i}$, and let $C^{\prime}=\bigcup_{i} \widehat{C}_{i}$. Then $C^{\prime}$ is a quasi-embedded GFL. Now the three terms in the inequality (15) are precisely the three terms in (17).

If some of the $C_{i}$ 's are trivial cylinders, then use the inequality (16), where $C^{\prime}$ is defined as above for the nontrivial components. 
Corollary 2.2 Let $C$ be a $G F L$ with $I(C)=1$. Assume that $J$ is generic and $(\phi, J)$ is $\operatorname{deg}(C)$-regular. Then $C$ has one nontrivial embedded component $C^{\prime}$; all other components are covers of trivial cylinders which do not intersect $C^{\prime}$.

That is, $C$ is a flow line, except that trivial cylinder components may be nontrivially covered instead of just repeated.

Proof We know that $C$ is a union of $d_{i}$-fold covers of distinct, connected, quasi-embedded GFL's $C_{i}$. Since $J$ is generic, $I^{\operatorname{vir}}\left(C_{i}\right) \geq 0$, with equality if and only if $C_{i}$ is trivial. So by the inequality (17), there is only one nontrivial $C_{i}$, and this has $d_{i}=1$ and does not intersect the trivial components.

A ( $k$ times) broken GFL is a sequence $\left(C_{0}, \ldots, C_{k}\right)$ of nontrivial GFL's such that for each $i=1, \ldots, k$, the outgoing ends of $C_{i}$ are identified with the incoming ends of $C_{i-1}$, such that two ends that are identified are at the same periodic orbit and with the same multiplicity. The broken GFL $\left(C_{0}, \ldots, C_{k}\right)$ is connected if the graph with one vertex for each component of each $C_{i}$, and an edge between two vertices when the corresponding components have ends identified, is connected. A component of the broken GFL $\left(C_{0}, \ldots, C_{k}\right)$ is a maximal connected broken GFL $\left(C_{0}^{\prime}, \ldots, C_{k}^{\prime}\right)$ such that each $C_{i}^{\prime}$ is a union of components of $C_{i}$. By Gromov compactness as in [9, Lem. 9.8], any sequence of nontrivial GFL's of the same topological type with bounded integral of $\omega$ has a subsequence which converges in an appropriate sense to a broken GFL.

\section{PFH of a twist on a cylinder}

Fix an integer $P$ and a positive integer $Q$. Fix real numbers $X_{1} \leq X_{2}$, and assume that neither is a rational number with denominator $\leq Q$. Let $\phi_{0}$ be the cylinder twist from equation (2). We identify the mapping torus

$$
\begin{aligned}
Y_{\phi_{0}} & \simeq S^{1} \times\left[X_{1}, X_{2}\right] \times S^{1}, \\
{[t,(x, y)] } & \mapsto(t, x, y-x t) .
\end{aligned}
$$

We now study the periodic Floer homology $H P_{*}(\phi, h)$, where $\phi$ is a perturbation of $\phi_{0}$ described below, and the homology class

$$
h:=Q\left[S^{1}\right] \times[p t] \times[p t]-P[p t] \times[p t] \times\left[S^{1}\right] .
$$

Note that $(\phi, h)$ is automatically monotone, with $\lambda=0$ in equation (8). We denote this periodic Floer homology by $H P_{*}\left(X_{1}, X_{2} ; P, Q\right)$. 


\subsection{Introduction and statement of results}

The perturbation We always write rational numbers in reduced form $p / q$ with $\operatorname{gcd}(p, q)=1$ and $q>0$. For each rational number $p / q \in\left[X_{1}, X_{2}\right]$, the map $\phi_{0}$ has a circle of period $q$ periodic orbits at $x=p / q$. As is familiar from KAM theory, we can perform a Hamiltonian perturbation of $\phi_{0}$ away from the boundary of $\left[X_{1}, X_{2}\right] \times S^{1}$ to obtain a map $\phi$ so that whenever $q \leq Q$, the above circle splits into two periodic orbits $e_{p / q}$ and $h_{p / q}$. The orbit $e_{p / q}$ is elliptic, and the orbit $h_{p / q}$ is hyperbolic with positive eigenvalues. For $p / q=0 / 1$ for example, $\phi_{0}$ and $\phi$ are the time-one maps of flows looking like this:
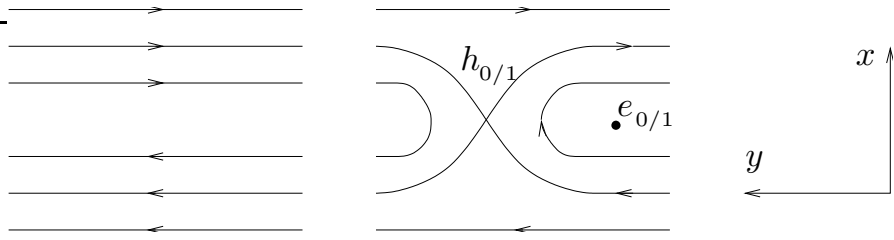

Throughout Section [3] we assume unless otherwise stated that:

- $\phi$ as above is chosen so that the $e_{p / q}$ 's and $h_{p / q}$ 's are the only irreducible periodic orbits of period $\leq Q$; these are nondegenerate; and the linearized return map of the elliptic orbit $e_{p / q}$ has eigenvalues $e^{ \pm 2 \pi i \theta}$ with $0<\theta<$ $1 / Q$.

- $\phi$ agrees with $\phi_{0}$ whenever $x$ is not within distance $\varepsilon$ of a rational number of denominator $\leq Q$, where $\varepsilon$ is sufficiently small with respect to $Q$.

- $J$ is a generic admissible almost complex structure on $\mathbb{R} \times Y_{\phi}$, such that the pair $(\phi, J)$ satisfies the local linearity condition in Section 2 with $d=Q$.

(In this example the last two conditions in the definition of $d$-regular are not needed to define PFH: bubbling of closed pseudoholomorphic curves in $\mathbb{R} \times Y$ cannot happen here because $\Omega$ is zero on $H_{2}\left(\mathbb{R} \times Y_{\phi}\right)$, and flow lines cannot approach the boundary by Lemma 3.11 below. In some arguments we will also drop the local linearity assumption.)

We denote the corresponding chain complex by $C P_{*}\left(X_{1}, X_{2} ; P, Q\right)$. We will see below that the homology of this chain complex, as well as most of the differential on it, does not depend on the choice of $\phi$ and $J$ as above.

The generators By (19), our chain complex is generated by admissible products of $e_{p / q}$ 's and $h_{p / q}$ 's with total numerator $P$ and total denominator $Q$. Recall that "admissible" means that no $h_{p / q}$ factor may be repeated. 
To each generator $\alpha$ we associate a left-turning convex polygonal path $\mathcal{P}(\alpha)$ in the plane as follows. Write $\alpha=\gamma_{1} \cdots \gamma_{k}$ where $\gamma_{i}=e_{p_{i} / q_{i}}$ or $\gamma_{i}=h_{p_{i} / q_{i}}$, and $\sum_{i=1}^{k}\left(p_{i}, q_{i}\right)=(P, Q)$. We order the factors so that $p_{i} / q_{i} \geq p_{j} / q_{j}$ for $i<j$. For $j=0, \ldots, k$, let $w_{j}=\sum_{i=0}^{j}\left(p_{i}, q_{i}\right)$, and define $\mathcal{P}(\alpha)$ to be the convex path in the plane consisting of straight line segments between the points $w_{j-1}$ and $w_{j}$ for $j=1, \ldots, k$, oriented so that the origin is the initial endpoint.

The homology Suppose $P / Q \in\left[X_{1}, X_{2}\right]$. We can uniquely write $(P, Q)=$ $v_{1}+v_{2}$, where the vector $v_{i}$ is in the upper half plane and has slope $X_{i}^{-1}$. Let $Z=Z\left(X_{1}, X_{2} ; P, Q\right) \subset \mathbb{R}^{2}$ denote the parallelogram with vertices $0, v_{1}, v_{2}$, and $(P, Q)$. We define

$$
E\left(X_{1}, X_{2} ; P, Q\right) \in C P_{*}\left(X_{1}, X_{2} ; P, Q\right)
$$

to be the unique generator $E$ such that $E$ is a product of elliptic factors, and the path $\mathcal{P}(E)$ traverses the right half of the boundary of the convex hull of the set of lattice points in the parallelogram $Z$. For example, if $-1 / 4<X_{1}<-1 / 5$ and $4 / 3<X_{2}<3 / 2$, then

$$
E\left(X_{1}, X_{2} ; 4,11\right)=e_{4 / 3} e_{1} e_{0}^{2} e_{-1 / 5},
$$

as shown by the following picture:

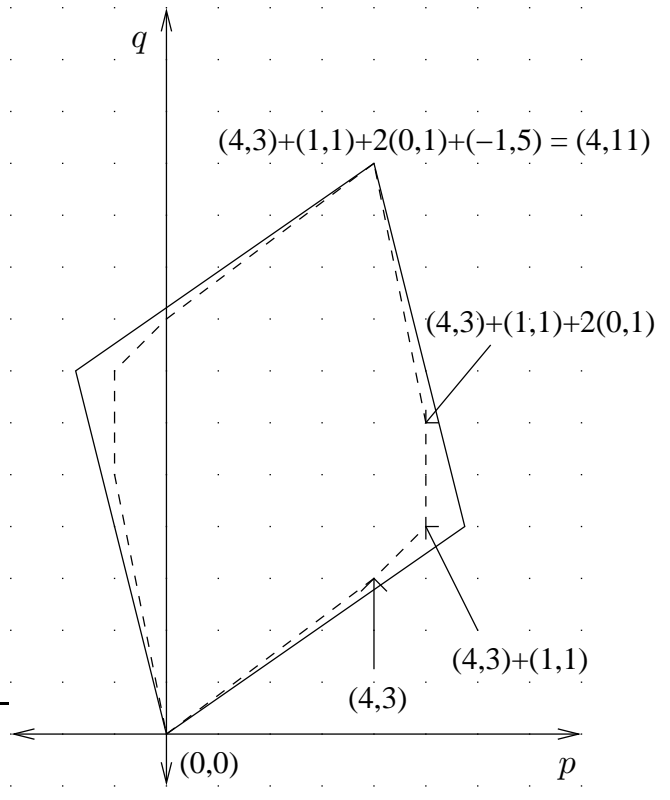


Theorem 3.1 (PFH of a twist on a cylinder)

$$
H P_{*}\left(X_{1}, X_{2} ; P, Q\right) \simeq\left\{\begin{array}{cl}
H_{*}\left(S^{1} ; \mathbb{Z} / 2\right) & \text { if } P / Q \in\left[X_{1}, X_{2}\right] \\
0 & \text { if } P / Q \notin\left[X_{1}, X_{2}\right] .
\end{array}\right.
$$

If $P / Q \in\left[X_{1}, X_{2}\right]$, then $H P_{*}\left(X_{1}, X_{2} ; P, Q\right)$ is generated by:

- $E\left(X_{1}, X_{2} ; P, Q\right)$, and

- any generator obtained from $E\left(X_{1}, X_{2} ; P, Q\right)$ by replacing one of the $e_{p / q}$ factors by $h_{p / q}$; all such generators are all homologous.

Combinatorial formulas Theorem 3.1 is proved in Section 3.9 First, in Section 3.2-Section 3.8 we derive combinatorial formulas for the relative index and most of the differential $\delta$ in the chain complex $C P_{*}\left(X_{1}, X_{2} ; P, Q\right)$. To state these, let $\alpha$ and $\beta$ be generators. We will see in Section 3.2 that the relative index $I(\alpha, \beta ; Z) \in \mathbb{Z}$ does not depend on $Z$, so we write it as $I(\alpha, \beta)$, thus obtaining a relative $\mathbb{Z}$-grading on $C P_{*}\left(X_{1}, X_{2} ; P, Q\right)$. We then have:

Proposition 3.2 (Index formula) If there are $a$ elliptic factors in $\alpha$ and $b$ elliptic factors in $\beta$, then

$$
I(\alpha, \beta)=b-a+2 \int_{\mathcal{P}(\beta)-\mathcal{P}(\alpha)} p d q .
$$

Remark 3.3 Here $\int_{\mathcal{P}(\beta)-\mathcal{P}(\alpha)} p d q$ denotes the signed area enclosed by the paths $-\mathcal{P}(\alpha)$ and $\mathcal{P}(\beta)$. Pick's theorem confirms that this is a half-integer. If $\mathcal{P}(\alpha)$ never crosses to the right of $\mathcal{P}(\beta)$ and if $\alpha$ and $\beta$ contain no hyperbolic factors, then $I(\alpha, \beta)$ equals twice the number of lattice points in the region enclosed by $\mathcal{P}(\alpha)$ and $\mathcal{P}(\beta)$, not including the lattice points in $\mathcal{P}(\alpha)$.

If $\mathcal{P}_{1}$ and $\mathcal{P}_{2}$ are paths in the $(p, q)$-plane, let $\mathcal{P}_{1} \mathcal{P}_{2}$ denote the concatenation of $\mathcal{P}_{1}$ with the appropriate translate of $\mathcal{P}_{2}$.

Definition 3.4 We say that $\alpha$ is obtained from $\beta$ by rounding a corner if there exist orbit sets $\gamma_{1}, \gamma_{2}, \alpha^{\prime}, \beta^{\prime}$ such that:

(a) $\alpha=\gamma_{1} \alpha^{\prime} \gamma_{2}$ and $\beta=\gamma_{1} \beta^{\prime} \gamma_{2}$, where $\mathcal{P}(\alpha)=\mathcal{P}\left(\gamma_{1}\right) \mathcal{P}\left(\alpha^{\prime}\right) \mathcal{P}\left(\gamma_{2}\right)$ and $\mathcal{P}(\beta)=$ $\mathcal{P}\left(\gamma_{1}\right) \mathcal{P}\left(\beta^{\prime}\right) \mathcal{P}\left(\gamma_{2}\right)$.

(b) The path $\mathcal{P}\left(\alpha^{\prime}\right)$ does not cross to the right of $\mathcal{P}\left(\beta^{\prime}\right)$, and only intersects it at the two endpoints.

(c) There are no lattice points in between $\mathcal{P}\left(\alpha^{\prime}\right)$ and $\mathcal{P}\left(\beta^{\prime}\right)$. 
(d) $\beta^{\prime}$ has two factors; either $\beta^{\prime}$ has one hyperbolic factor and $\alpha^{\prime}$ has none, or $\beta^{\prime}$ has two hyperbolic factors and $\alpha^{\prime}$ has one.

We say that $\alpha$ is obtained from $\beta$ by double rounding if (a), (b), and (c) above hold, together with:

$\left(d^{\prime}\right) \quad \beta^{\prime}$ has three factors, all hyperbolic, and $\alpha^{\prime}$ has no hyperbolic factors.

Theorem 3.5 (The differential) Let $\alpha, \beta$ be generators of $C P_{*}\left(X_{1}, X_{2} ; P, Q\right)$. Then for any $\phi$ and $J$ as in Section 3.1.

(a) If $\langle\delta \alpha, \beta\rangle=1$, then $\alpha$ is obtained from $\beta$ by rounding a corner or by double rounding.

(b) If $\alpha$ is obtained from $\beta$ by rounding a corner, then $\langle\delta \alpha, \beta\rangle=1$.

Here $\langle\delta \alpha, \beta\rangle \in \mathbb{Z} / 2$ denotes the coefficient of $\beta$ in $\delta \alpha$.

Remark 3.6 If we drop the local linearity requirement (in which case more analytic work would be needed to show that $\mathrm{PFH}$ is well-defined) and consider $(\phi, J)$ close in an appropriate norm to $\left(\phi_{0}, J_{0}\right)$, where $J_{0}$ is a certain $S^{1}$ invariant almost complex structure on $Y_{\phi_{0}}$, then we can arrange that there are no flow lines from $\alpha$ to $\beta$ when $\alpha$ is obtained from $\beta$ by double rounding, see Appendix A. In any case, we will see in Section 3.9 that differential coefficients involving double rounding have no effect on the homology.

\subsection{Calculating the relative index}

Let $\alpha$ and $\beta$ be generators of $C P_{*}\left(X_{1}, X_{2} ; P, Q\right)$ and let $Z \in H_{2}(Y ; \alpha, \beta)$. We now prove Proposition 3.2 computing the relative index $I(\alpha, \beta ; Z)$.

The bundle $V \simeq T\left(\left[X_{1}, X_{2}\right] \times S^{1}\right)$ over $Y_{\phi} \simeq S^{1} \times\left[X_{1}, X_{2}\right] \times S^{1}$ has a canonical trivialization up to homotopy, giving rise to a natural trivialization $\tau$ over each periodic orbit, which we use for the rest of Section 3.2

Because the trivialization $\tau$ comes from a global trivialization of $V$,

$$
c_{\tau}(Z)=0 .
$$

We now compute the Conley-Zehnder indices as in 9, Section 2.3]. The elliptic orbit $e_{p / q}$ has slightly negative monodromy angle $\theta$ with respect to $\tau$, so

$$
\mu_{\tau}\left(e_{p / q}^{k}\right)=2\lfloor k \cdot \theta\rfloor+1=-1
$$


for $k q \leq Q$. The hyperbolic orbit $h_{p / q}$ has positive eigenvalues, and the eigenspaces do not rotate with respect to $\tau$, so

$$
\mu_{\tau}\left(h_{p / q}\right)=0 .
$$

We next observe that $Q_{\tau}(Z)$ depends only on $\alpha$ and $\beta$, by [9, Lem. 2.5], since $H_{2}(Y)$ is generated by a $(y, t)$-torus, which has algebraic intersection number zero with every periodic orbit. So denote the integer $Q_{\tau}(Z)$ by $Q_{\tau}(\alpha, \beta)$; by (20), we can likewise write $I(\alpha, \beta):=I(\alpha, \beta ; Z)$.

Lemma 3.7 If $\alpha$ and $\beta$ are generators of $C P_{*}\left(X_{1}, X_{2} ; P, Q\right)$ then

$$
Q_{\tau}(\alpha, \beta)=2 \int_{\mathcal{P}(\beta)-\mathcal{P}(\alpha)} p d q
$$

Proof Write $\alpha=\gamma_{1} \cdots \gamma_{k}$ and $\beta=\gamma_{1}^{\prime} \cdots \gamma_{l}^{\prime}$, where $\gamma_{i}=e_{p_{i} / q_{i}}$ or $\gamma_{i}=h_{p_{i} / q_{i}}$, and $\gamma_{i}^{\prime}=e_{p_{i}^{\prime} / q_{i}^{\prime}}$ or $\gamma_{i}^{\prime}=h_{p_{i}^{\prime} / q_{i}^{\prime}}$. We order the factors so that $p_{i} / q_{i} \geq p_{j} / q_{j}$ and $p_{i}^{\prime} / q_{i}^{\prime} \geq p_{j}^{\prime} / q_{j}^{\prime}$ for $i<j$. We claim that

$$
Q_{\tau}(\alpha, \beta)=-\sum_{i<j} \operatorname{det}\left(\begin{array}{cc}
p_{i} & p_{j} \\
q_{i} & q_{j}
\end{array}\right)+\sum_{i<j} \operatorname{det}\left(\begin{array}{cc}
p_{i}^{\prime} & p_{j}^{\prime} \\
q_{i}^{\prime} & q_{j}^{\prime}
\end{array}\right) .
$$

By [9. Eq. (13)], if $S \subset[0,1] \times Y$ is an embedded (except at the boundary) representative of a relative homology class $Z \in H_{2}(Y ; \alpha, \beta)$, then

$$
Q_{\tau}(\alpha, \beta)=c_{1}(N, \tau)-w_{\tau}(S)
$$

where $N$ denotes the normal bundle to $S$ and $c_{1}(N, \tau)$ its relative first Chern class. To prove (24), we apply (25) to the following surface $S$. We regard $S$ as a movie of curves in $Y$ parametrized by $s \in[0,1]$. For $s$ close to 1 , the slice $S \cap(\{s\} \times Y)$ consists of one circle in the $x=p / q$ torus for each factor $\gamma_{p / q}$ in $\alpha$, parallel to the periodic orbits $e_{p / q}$ and $h_{p / q}$. As $s$ decreases to $2 / 3$, we translate all of the circles in the $x$ direction into a single $(y, t)$-torus. Around $s=2 / 3$, we perform "negative surgeries" so that at $s=1 / 2$, we have an embedded union of circles in a single $(y, t)$-torus. Near a negative surgery, the $s>2 / 3$ and $s<2 / 3$ slices look like the left and right sides of the following picture:

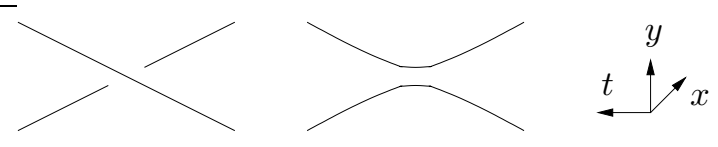


For $s$ between $1 / 2$ and 0 we perform an opposite process, doing "positive surgeries" at $s=1 / 3$, thereby obtaining, for $s$ close to zero, one circle in the $x=p / q$ torus for each factor $\gamma_{p / q}$ in $\beta$. We can assume that on the complement of the surgery points, our circles never point in the $y$ direction.

By construction, $w_{\tau}(S)=0$. To compute $c_{1}(N, \tau)$, let $\psi:=\pi_{N} \partial_{y} \in \Gamma(N)$. Since $\psi$ has winding number zero with respect to $\tau$ on $\partial S$, the integer $c_{1}(N, \tau)$ equals the algebraic count of zeroes of $\psi$. The section $\psi$ vanishes only at the surgery points. The number of negative surgeries equals the first sum of determinants (without the minus sign) on the right side of equation (24), and the number of positive surgeries equals the second sum. It is an exercise to check that $\psi$ has -1 zero at each negative surgery point and +1 zero at each positive surgery point. This proves (24).

To deduce (23), observe that the right hand sides of (23) and (24) change by the same amount if we replace two consecutive edges in $\mathcal{P}(\alpha)$ or $\mathcal{P}(\beta)$ by their sum. This inductively reduces to the trivial case where $\mathcal{P}(\alpha)$ and $\mathcal{P}(\beta)$ are both straight lines.

Proof of Proposition 3.2 This follows immediately from equations (20), (21), (22) and (23).

\subsection{Some constraints on index 1 flow lines}

Lemma 3.8 (Complexity) Let $\alpha, \beta \in C P_{*}\left(X_{1}, X_{2} ; P, Q\right)$ be generators with $I(\alpha, \beta)=1$, let $C \in \mathcal{M}(\alpha, \beta)$, and $C^{\prime}:=C \backslash\{$ trivial cylinders $\}$. Then:

(a) $C^{\prime}$ is connected and has genus zero.

(b) If $e_{-}\left(C^{\prime}\right)$ denotes the number of incoming elliptic ends of $C^{\prime}$, and $h\left(C^{\prime}\right)$ denotes the number of hyperbolic ends of $C^{\prime}$, then

$$
2 e_{-}\left(C^{\prime}\right)+h\left(C^{\prime}\right)=3 \text {. }
$$

(c) Any incoming elliptic end of $C^{\prime}$ has multiplicity one. By contrast, $C^{\prime}$ has only one outgoing end at any given elliptic orbit.

Proof We have $1 \leq \operatorname{dim}\left(\mathcal{M}_{C^{\prime}}\right) \leq I^{\operatorname{vir}}\left(C^{\prime}\right) \leq I\left(C^{\prime}\right) \leq I(C)=1$, since $\mathbb{R}$ acts nontrivially on $\mathcal{M}_{C^{\prime}}$ by translation in $\mathbb{R} \times Y$, and using the inequalities (12), (15) and (16). Then $C^{\prime}$ is connected, since otherwise $\operatorname{dim}\left(\mathcal{M}_{C^{\prime}}\right) \geq 2$.

Let $e_{+}\left(C^{\prime}\right)$ denote the number of outgoing elliptic ends of $C^{\prime}$. By (11), (20), (21), and (22),

$$
I^{\operatorname{vir}}\left(C^{\prime}\right)=-\chi\left(C^{\prime}\right)-e_{+}\left(C^{\prime}\right)+e_{-}\left(C^{\prime}\right) .
$$


Putting in $I^{\mathrm{vir}}=1$ and $\chi=2-2 g-e_{-}-e_{+}-h$, we obtain

$$
2 g\left(C^{\prime}\right)+2 e_{-}\left(C^{\prime}\right)+h\left(C^{\prime}\right)=3 .
$$

If $g\left(C^{\prime}\right)>0$, then (26) implies that $C^{\prime} \in \mathcal{M}\left(\alpha^{\prime}, \beta^{\prime}\right)$ where $\alpha^{\prime}$ is a product of elliptic factors and $\beta^{\prime}=h_{p / q}$ for some $p / q$. Then by Proposition 3.2, $I\left(\alpha^{\prime}, \beta^{\prime}\right)<$ 0 , because the area term is $\leq 0$ by convexity. This contradicts $I\left(C^{\prime}\right)=1$.

The above proves parts (a) and (b) of the lemma. Part (c) follows from the discussion of incoming and outgoing partitions in Section 2] see equation (10).

Lemma 3.9 (Trivial cylinders) Let $\alpha \gamma, \beta \gamma \in C P_{*}\left(X_{1}, X_{2} ; P, Q\right)$ be generators, where $\alpha$ and $\beta$ have no periodic orbits in common. Assume $I(\alpha, \beta)=$ $I(\alpha \gamma, \beta \gamma)=1$. Then attaching trivial cylinders over $\gamma$ gives a bijection

$$
\mathcal{M}(\alpha, \beta) \simeq \mathcal{M}(\alpha \gamma, \beta \gamma) \text {. }
$$

Proof By (16) and intersection positivity, if $I\left(C^{\prime}\right)=I\left(C^{\prime} \cup T\right)$ then $C^{\prime} \cap T=\emptyset$. So since $I(\alpha, \beta)=I(\alpha \gamma, \beta \gamma)$, it follows that attaching trivial cylinders gives a well defined map $\mathcal{M}(\alpha, \beta) \rightarrow \mathcal{M}(\alpha \gamma, \beta \gamma)$, and this is clearly injective.

Now this map must be surjective. For suppose there exists $C \in \mathcal{M}(\alpha \gamma, \beta \gamma)$ which does not contain trivial cylinders over all of $\gamma$. Let $C^{\prime}$ denote the nontrivial component of $C$. Then $C^{\prime}$ has both an incoming end and an outgoing end at some orbit $\rho$ in $\gamma$. If $\rho$ is hyperbolic, then $\rho$ is not in the generators $\alpha$ and $\beta$ (since $\alpha \gamma$ and $\beta \gamma$ are admissible orbit sets). If $\rho$ is elliptic, then by Lemma 3.8 (c), $C^{\prime}$ has an incoming end of multiplicity one there. Either way, ends of $C^{\prime}$ at $\gamma$ alone contribute at least 2 to the left hand side of equation (26). Hence Lemma 3.8(b) implies that $\beta=h_{p / q}$ for some $p / q$, and $\alpha$ is a product of elliptic factors. By Proposition 3.2. $I(\alpha, \beta)<0$, which is a contradiction.

Remark 3.10 The analogue of Lemma 3.9 for a single Dehn twist on a torus is false. We will see in Section 4.4 that in the notation of that section, there exists $C \in \mathcal{M}\left(e^{2}, e h ; Z\right)$ with no trivial cylinders even though

$$
I\left(e^{2}, e h ; Z\right)=I(e, h ; Z)=1 .
$$

\subsection{The local energy inequality}

The general case Let $\phi$ be a general symplectomorphism of $\Sigma$. Suppose $\xi \subset \Sigma$ is an invariant circle, i.e. $\phi(\xi)=\xi$, such that $\left.\phi\right|_{\xi}$ is smoothly conjugate 
to a rotation. Let $T:=Y_{\left.\phi\right|_{\xi}}$ and let $N \subset \Sigma$ be a tubular neighborhood of $\xi$. We choose local coordinates $x \in(-\epsilon, \epsilon)$ and $y, t \in \mathbb{R} / \mathbb{Z}$ on $Y_{\left.\phi\right|_{N}}$ such that $T=\{x=0\}$, the mapping torus flow at $x=0$ is $R=\partial_{t}-\theta \partial_{y}$ with $\theta \in \mathbb{R}$ fixed, and $\omega\left(\partial_{x}, \partial_{y}\right)>0$. Let $J$ be an admissible almost complex structure on $\mathbb{R} \times Y_{\phi}$.

Let $C$ be a generalized flow line transverse to $\mathbb{R} \times T$. Orient $C \cap(\mathbb{R} \times T)$ so that if $p \in C \cap(\mathbb{R} \times T)$ and $\{v, w\}$ is an oriented basis of $T_{p} C$ with $v \in T_{p}(C \cap(\mathbb{R} \times T))$ positively oriented, then $w$ has negative $\partial_{x}$ component.

Lemma 3.11 The homology class of the slice

$$
(p, q):=[C \cap(\mathbb{R} \times T)] \in H_{1}\left(S_{y}^{1} \times S_{t}^{1}\right)
$$

satisfies $p+\theta q \geq 0$, with equality iff $C \cap(\mathbb{R} \times T)=\emptyset$.

Proof Each component of $C \cap(\mathbb{R} \times T)$ can be described by an oriented parametrized curve $\gamma(\tau)$. Let $a(y, t)$ denote the component of $\partial_{x}$ in $J \partial_{y}$ at $x=0$. Since $J$ is $\Omega$-tame, $a(y, t)<0$. At $x=0$, since $J\left(\partial_{t}-\theta \partial_{y}\right)=-\partial_{s}$, the component of $\partial_{x}$ in $J \partial_{s}$ is zero, and the component of $\partial_{x}$ in $J \partial_{t}$ is $\theta a(y, t)$. Since $C$ is $J$-holomorphic, $J\left(\gamma^{\prime}(\tau)\right) \in T C$, and by transversality and our sign conventions, this has negative $\partial_{x}$ component. That is, if $\gamma(\tau)=(s(\tau), y(\tau), t(\tau))$, then

$$
\frac{d y}{d \tau}+\theta \frac{d t}{d \tau}>0
$$

This inequality can also be understood as intersection positivity of $C$ with the $J$-holomorphic foliation of $\mathbb{R} \times T$ by $\mathbb{R}$ cross the mapping torus flow. Integrating this inequality over $\tau$, we conclude that the homology class $\left(p^{\prime}, q^{\prime}\right)$ of $\gamma$ satisfies $p^{\prime}+\theta q^{\prime}>0$.

The cylinder twist case For a Dehn twist on a cylinder, the local energy inequality of Lemma 3.11 has the following interpretation.

Proposition 3.12 Let $\alpha$ and $\beta$ be generators of $C P_{*}\left(X_{1}, X_{2} ; P, Q\right)$. Suppose that the path $\mathcal{P}(\alpha)$ crosses to the right of $\mathcal{P}(\beta)$. Then for any $\phi$ as in Section 3.1 and any admissible $J$, there are no flow lines from $\alpha$ to $\beta$.

Proof Suppose there exist flow lines from $\alpha$ to $\beta$ for arbitrarily small values of the constant $\varepsilon$ in Section [3.1 Let $p_{i}, q_{i}, p_{j}^{\prime}, q_{j}^{\prime}$ be defined as in the proof of Lemma 3.7. We first claim that for each $x_{0} \in \mathbb{R}$, if $\varepsilon$ is sufficiently small then

$$
0 \leq \sum_{p_{i} / q_{i}>x_{0}}\left(q_{i} x_{0}-p_{i}\right)-\sum_{p_{j}^{\prime} / q_{j}^{\prime}>x_{0}}\left(q_{j}^{\prime} x_{0}-p_{j}^{\prime}\right)
$$


To prove (27), by continuity we may assume that $x_{0}$ is not a rational number of denominator $\leq Q$. We can then assume that $\varepsilon$ is sufficiently small that $\phi$ agrees with $\phi_{0}$ for $x$ near $x_{0}$. By continuity again, we may assume that $C$ intersects the locus $\left\{x=x_{0}\right\}$ transversely. Then (27) follows from Lemma 3.11] since

$$
\left[C \cap\left\{x=x_{0}\right\}\right]=\sum_{p_{i} / q_{i}>x_{0}}\left(-p_{i}, q_{i}\right)-\sum_{p_{j}^{\prime} / q_{j}^{\prime}>x_{0}}\left(-p_{j}^{\prime}, q_{j}^{\prime}\right) \in H_{1}\left(S_{y}^{1} \times S_{t}^{1}\right) .
$$

Now suppose $(a, b)$ and $(c, d)$ are two intersections of the paths $\mathcal{P}(\alpha)$ and $\mathcal{P}(\beta)$, between which $\mathcal{P}(\alpha)$ is to the right of $\mathcal{P}(\beta)$. Since both paths move in the positive $q$ direction we may assume that $b<d$. Let $(p, q)$ and $\left(p^{\prime}, q^{\prime}\right)$ be points on $\mathcal{P}(\alpha)$ and $\mathcal{P}(\beta)$ respectively between $(a, b)$ and $(c, d)$ which are as far as possible from the line through $(a, b)$ and $(c, d)$. Then $(p, q)$ is farther from this line than $\left(p^{\prime}, q^{\prime}\right)$, so

$$
\operatorname{det}\left(\begin{array}{ll}
p-p^{\prime} & c-a \\
q-q^{\prime} & d-b
\end{array}\right)>0
$$

If we let $x_{0}:=(c-a) /(d-b)$ then by equation (27) we have

$$
0 \leq\left(q x_{0}-p\right)-\left(q^{\prime} x_{0}-p^{\prime}\right) .
$$

This inequality contradicts $b<d$ and (29).

Lemma 3.13 If $\left[X_{1}, X_{2}\right] \subset\left[X_{1}^{\prime}, X_{2}^{\prime}\right]$, then for compatible choices of $J$ and $\phi$ as in Section 3.1, the differential $\delta$ commutes with the inclusion

$$
C P_{*}\left(X_{1}, X_{2} ; P, Q\right) \longrightarrow C P_{*}\left(X_{1}^{\prime}, X_{2}^{\prime} ; P, Q\right) .
$$

Proof If $\alpha, \beta \in C P_{*}\left(X_{1}, X_{2} ; P, Q\right)$, then any flow line from $\alpha$ to $\beta$ in $S^{1} \times$ $\left[X_{1}^{\prime}, X_{2}^{\prime}\right] \times S^{1}$ is supported in $S^{1} \times\left[X_{1}, X_{2}\right] \times S^{1}$, by Lemma 3.11.

\subsection{Vanishing of some differential coefficients}

Lemma 3.14 If $\lambda q \leq Q$, then for any $J$ and $\phi$ as in Section 3.1.

$$
\left\langle\delta\left(e_{p / q}^{\lambda-1} h_{p / q}\right), e_{p / q}^{\lambda}\right\rangle=0 .
$$

Proof The trivial cylinder lemma 3.9 shows that

$$
\left\langle\delta\left(e_{p / q}^{\lambda-1} h_{p / q}\right), e_{p / q}^{\lambda}\right\rangle=\left\langle\delta h_{p / q}, e_{p / q}\right\rangle .
$$


By Lemma 3.8(a), any flow line from $h_{p / q}$ to $e_{p / q}$ is a cylinder. Now for the unperturbed map $\phi_{0}$ with an almost complex structure such as $J_{0}$ in Appendix A any pseudoholomorphic cylinder with incoming and outgoing ends at $x=p / q$ is trivial, as in [9, Prop. 9.1]. Then a standard argument in Morse-Bott theory shows that for $\left(\phi^{\prime}, J^{\prime}\right)$ close to $\left(\phi_{0}, J_{0}\right)$, there are two cylinders from $h_{p / q}$ to $e_{p / q}$. These cylinders are both embedded by the adjunction formula (13), because all terms in (13) other than $2 \delta(C)$ are automatically zero. As we deform $\left(\phi^{\prime}, J^{\prime}\right)$ to $(\phi, J)$, no broken GFL's from $h_{p / q}$ to $e_{p / q}$ appear during the deformation by Lemma 3.11. It follows by Gromov compactness as in [9, Lem. 9.8] that the mod 2 count of such cylinders remains zero.

Proof of Theorem [3.5(a) Suppose $\langle\delta \alpha, \beta\rangle \neq 0$.

Without loss of generality, $\mathcal{P}(\alpha)$ and $\mathcal{P}(\beta)$ have no initial edges in common. To see this, suppose that $\alpha$ and $\beta$ both contain factors of $e_{p / q}$ or $h_{p / q}$ but do not contain factors $e_{p^{\prime} / q^{\prime}}$ or $h_{p^{\prime} / q^{\prime}}$ with $p^{\prime} / q^{\prime}>p / q$. We claim that $\alpha$ and $\beta$ have a common factor of $e_{p / q}$ or $h_{p / q}$, so that we can remove it from both by the trivial cylinder lemma 3.9. Otherwise, either: (1) $\alpha$ contains an $h_{p / q}$ factor and $\beta$ contains an $e_{p / q}^{\lambda}$ factor, or (2) $\alpha$ contains an $e_{p / q}^{\lambda}$ factor and $\beta$ contains an $h_{p / q}$ factor. In case (1), we must have $\lambda=1$ by Lemma 3.8(b). By Lemma 3.11] if $C \in \mathcal{M}(\alpha, \beta)$, then $C \cap\{x=p / q-\varepsilon\}=\emptyset$, so

$$
\mathcal{M}(\alpha, \beta)=\mathcal{M}\left(\alpha / h_{p / q}, \beta / e_{p / q}\right) \times \mathcal{M}\left(h_{p / q}, e_{p / q}\right),
$$

so $\langle\delta \alpha, \beta\rangle=0$ by Lemma 3.14. In case (2), we have $\lambda=1$ by Proposition 3.12. Then as in case (1),

$$
\mathcal{M}(\alpha, \beta)=\mathcal{M}\left(\alpha / e_{p / q}, \beta / h_{p / q}\right) \times \mathcal{M}\left(e_{p / q}, h_{p / q}\right) .
$$

But $\mathcal{M}\left(e_{p / q}, h_{p / q}\right)=\emptyset$, since $I\left(e_{p / q}, h_{p / q}\right)=-1$, so $\langle\delta \alpha, \beta\rangle=0$.

Likewise, WLOG the paths $\mathcal{P}(\alpha)$ and $\mathcal{P}(\beta)$ have no final edges in common.

By Proposition 3.12, $\mathcal{P}(\alpha)$ does not cross to the right of $\mathcal{P}(\beta)$. Moreover, the paths $\mathcal{P}(\alpha)$ and $\mathcal{P}(\beta)$ do not intersect except at their endpoints. For if the paths intersect elsewhere, then the intersections must be lattice points or segments bounded by lattice points. These intersections cut $\mathcal{P}(\beta)$ into at least two pieces. By convexity, each piece has at least two edges, and the initial and final edges of each piece do not have parallel edges in $\mathcal{P}(\alpha)$. It follows that, aside from possible trivial cylinders, flow lines from $\alpha$ to $\beta$ have at least four incoming ends, contradicting Lemma 3.8(b).

Thus conditions (a) and (b) in Definition 3.4 hold. Since $I(\alpha, \beta)=1$, conditions (c) and either (d) or $\left(\mathrm{d}^{\prime}\right)$ in Definition 3.4 follow from the index formula of Proposition 3.2 and Remark 3.3 


\subsection{Invariance of rounding coefficients}

Suppose that $\alpha$ is obtained from $\beta$ by rounding a corner, and that $\alpha$ and $\beta$ have no periodic orbits in common. By Lemma 3.8 the flow lines $C \in \mathcal{M}(\alpha, \beta)$ counted by the differential coefficient $\langle\delta \alpha, \beta\rangle$ satisfy the following conditions:

(i) $C$ is connected and has genus zero.

(ii) For each $p / q$, the flow line $C$ has at most one outgoing end of any multiplicity at $e_{p / q}$.

Now suppose that $\phi$ is as in Section 3.1 and that $J$ is admissible and generic, but not necessarily local linear. In this case we define $\langle\delta \alpha, \beta\rangle$ to be the mod 2 count of flow lines $C \in \mathcal{M}(\alpha, \beta) / \mathbb{R}$ satisfying conditions (i) and (ii) above. Note that such $C$ are isolated by the index calculation in Section 3.3 .

Lemma 3.15 Let $\alpha$ and $\beta$ be generators of $C_{*}\left(X_{1}, X_{2} ; P^{\prime}, Q^{\prime}\right)$ with no periodic orbits in common, where $\alpha$ is obtained from $\beta$ by rounding a corner. Suppose $\phi$ is as in Section 3.1 with $Q \geq Q^{\prime}$ and $J$ is admissible and generic. Then:

(a) $\langle\delta \alpha, \beta\rangle$ as above is well defined, i.e. the set of $C \in \mathcal{M}(\alpha, \beta) / \mathbb{R}$ satisfying (i) and (ii) is finite.

(b) $\langle\delta \alpha, \beta\rangle$ does not depend on $\phi, J$, or $Q$ as above.

Proof (a) By Gromov compactness as in [9, Lem. 9.8], it is enough to show that there does not exist a $k$-times broken GFL from $\alpha$ to $\beta$ with $k \geq 1$ satisfying conditions (i) and (ii).

Suppose that $C=\left(C_{0}, \ldots, C_{k}\right)$ is such a broken GFL. Let $C_{i, j}$ denote the components of $C_{i}$. We have $I^{\mathrm{vir}}\left(C_{i, j}\right)=-\chi\left(C_{i, j}\right)+\mu_{\tau}^{0}\left(C_{i, j}\right)$, which together with the genus zero condition from (i) and equations (21) and (22) implies that

$$
2 e_{-}\left(C_{i, j}\right)+h\left(C_{i, j}\right)=2+I^{\mathrm{vir}}\left(C_{i, j}\right) .
$$

Also $\sum_{i, j} I^{\operatorname{vir}}\left(C_{i, j}\right)=1$.

Each $C_{i, j}$ must satisfy $I^{\mathrm{vir}}\left(C_{i, j}\right) \geq 0$. Otherwise (30) implies that $C_{i, j}$ has only one incoming end at some $h_{p / q}$, and all outgoing ends elliptic. In particular the quasi-embedded curve underlying $C_{i, j}$ lives in a moduli space of expected dimension $\leq-1$ (even before modding out by the $\mathbb{R}$ action), which is impossible for generic $J$ (or even during a generic one-parameter deformation).

Therefore one of the $C_{i, j}$ 's has $I^{\mathrm{vir}}=1$, while all other $C_{i, j}$ 's have $I^{\mathrm{vir}}=0$. Also, by Proposition 3.12 all but one of the $C_{i, j}$ 's goes between orbit sets with 
the same polygonal path, and hence by Lemma 3.11 maps to a neighborhood of $\theta=p / q$ for some $p / q$ depending on the $C_{i, j}$. Let $\widehat{C}$ denote the remaining $C_{i, j}$; this has two incoming ends corresponding to the edges of the corner being rounded.

We claim that $I^{\mathrm{vir}}(\widehat{C})=0$. Suppose not, so that all $C_{i, j} \neq \widehat{C}$ have $I^{\mathrm{vir}}=0$. Then $\widehat{C}=C_{k}$. (If $\widehat{C}=C_{l}$ with $l<k$, then each $C_{i, j}$ with $i>l$ is a cylinder. By 9. Prop. 9.1], any cylinder with the same ends is trivial. Thus one of these cylinders must have distinct ends and hence $I^{\mathrm{vir}} \neq 0$.) Since $C$ has genus zero, downward induction on $i$ shows that each $C_{i, j}$ with $i<k$ has only one incoming end. It follows by equation (30) that each nontrivial $C_{i, j}$ with $i<k$ has more than one outgoing end. Since $k>1$, this leads to a contradiction of condition (ii).

So $I^{\text {vir }}(\widehat{C})=0$, and since $\widehat{C}$ has incoming ends of multiplicity one, $\widehat{C}$ is not multiply covered and hence does not exist for generic $J$.

(b) Consider a generic one-parameter deformation of $\phi$ and $J$. By Gromov compactness as in 9. Lem. 9.8], $\langle\delta \alpha, \beta\rangle$ can change during the deformation only at those times when there exists a broken GFL $C$ from $\alpha$ to $\beta$ satisfying (i) and (ii). The classification of such broken GFL's from part (a) is still valid, except that now $\widehat{C}$ as above may exist at isolated times in a generic one-parameter family. By equation (30), the two incoming ends of $\widehat{C}$ are hyperbolic, while all incoming ends of $\widehat{C}$ are elliptic. We claim that $k=1$, that $\widehat{C}=C_{0}$ or $\widehat{C}=C_{1}$, and that all components of $C_{1}$ or $C_{0}$ respectively are trivial except for one cylinder whose incoming end is at $e_{p / q}$ and whose outgoing end is at $h_{p / q}$ for some $p / q$. This follows from (i), (ii), and (30) using induction over the components of $C$. As in Lemma 3.14 there are two cancelling cylinders from $e_{p / q}$ to $h_{p / q}$. Therefore standard gluing arguments as in 6, 14, 23. show that the mod 2 count $\langle\delta \alpha, \beta\rangle$ does not change in this bifurcation.

Finally, $\langle\delta \alpha, \beta\rangle$ is independent of $Q \geq Q^{\prime}$, because if $\phi$ satisfies the conditions in Section 3.1 for a given value of $Q$, then it also does for any smaller value of $Q$.

\section{7 $\quad S L_{2} \mathbb{Z}$ symmetry}

We now observe a useful symmetry of our chain complex. Let $A=\left(\begin{array}{ll}a & b \\ c & d\end{array}\right) \in$ $S L_{2} \mathbb{Z}$. Define $I_{A}:=\{x \in \mathbb{R} \mid c x+d>0\}$. If $(p, q)$ is in the upper half plane and $p / q \in I_{A}$, then $A(p, q)$ is also in the upper half plane. So if $\left[X_{1}, X_{2}\right] \subset I_{A}$, 
then there is a well-defined linear map

$$
\Psi_{A}: C P_{*}\left(X_{1}, X_{2} ; P, Q\right) \longrightarrow C P_{*}\left(\frac{a X_{1}+b}{c X_{1}+d}, \frac{a X_{2}+b}{c X_{2}+d} ; a P+b Q, c P+d Q\right)
$$

that replaces every occurrence of $e_{p / q}$ with $e_{\frac{a p+b q}{c p+d q}}$ and $h_{p / q}$ with $h_{\frac{a p+b q}{c p+d q}}$. If $\alpha$ is obtained from $\beta$ by rounding a corner, then $\Psi_{A}(\alpha)$ is obtained from $\Psi_{A}(\beta)$ by rounding a corner.

Lemma 3.16 Under the assumptions of Lemma 3.15, let $A \in S L_{2} \mathbb{Z}$, suppose $\left[X_{1}, X_{2}\right] \subset I_{A}$, and suppose $c P^{\prime}+d Q^{\prime} \leq Q$. Then

$$
\left\langle\delta \Psi_{A}(\alpha), \Psi_{A}(\beta)\right\rangle=\langle\delta \alpha, \beta\rangle .
$$

Proof Using the coordinates (18), we define an orientation-preserving diffeomorphism of mapping tori

$$
\begin{aligned}
\psi_{A}: S^{1} \times\left[X_{1}, X_{2}\right] \times S^{1} & \longrightarrow S^{1} \times\left[c X_{1}+d, c X_{2}+d\right] \times S^{1}, \\
(t, x, y) & \longmapsto\left(-c y+d t, \frac{a x+b}{c x+d}, a y-b t\right) .
\end{aligned}
$$

Then $\psi_{A}$ sends the mapping torus flow $R=\partial_{t}-x \partial_{y}$ for $\phi_{0}$ to a positive multiple of itself, namely

$$
\left(\psi_{A}\right)_{*} R=\frac{R}{a-c x} .
$$

In particular, $\psi_{A}$ sends the circle of periodic orbits at $x=p / q$ to the circle of periodic orbits at $x=(a p+b q) /(c p+d q)$. Thus $\psi_{A}$ pulls back a pair $\left(\phi^{\prime}, J^{\prime}\right)$ for $X_{1}, X_{2}, Q$ from an admissible pair $(\phi, J)$ for $\left(a X_{1}+b\right) /\left(c X_{1}+d\right)$, $\left(a X_{2}+b\right) /\left(c X_{2}+d\right)$, and $c P+d Q$. But $J^{\prime}$ is not admissible, because $J^{\prime} \partial_{s}$ is only a positive multiple of $R$, and $J^{\prime}$ is $\Psi_{A}^{*} \Omega$-tame but not necessarily $\Omega$-tame.

In the coordinates (18) we have

$$
\Omega=d s \wedge d t+d x \wedge d y+x d x \wedge d t,
$$

from which we compute that

$$
\Omega \wedge \Psi_{A}^{*} \Omega=\frac{(c x+d)^{4}+1}{(c x+d)^{3}} d s \wedge d t \wedge d x \wedge d y>0 .
$$

Therefore linear interpolation defines a path of symplectic forms from $\Omega$ to $\Psi_{A}^{*} \Omega$. Let $J^{\prime \prime}$ be an admissible almost complex structure for $X_{1}, X_{2}, Q$. With respect to this path of symplectic forms, we can find a path of tame almost complex structures from $J^{\prime \prime}$ to $J^{\prime}$ that are $\mathbb{R}$-invariant and that send the Reeb flow to a positive multiple of itself. Then a compactness argument as in Lemma 3.15 shows that the differential coefficient $\langle\delta \alpha, \beta\rangle$ stays well defined and does not change during the deformation, and hence equals $\left\langle\delta \Psi_{A}(\alpha), \Psi_{A}(\beta)\right\rangle$. 


\subsection{Nonvanishing of some differential coefficients}

Lemma 3.17 Let $Q^{\prime} \leq Q$, and let $\alpha$ and $\beta$ be generators of $C_{*}\left(X_{1}, X_{2} ; P^{\prime}, Q^{\prime}\right)$ with no periodic orbits in common, where $\alpha$ is obtained from $\beta$ by rounding a corner. Then for any $\phi$ and $J$ as in Section 3.1, we have $\langle\delta \alpha, \beta\rangle=1$.

Proof More explicitly, we can write $\beta=e_{c / d} h_{a / b}$ or $\beta=h_{c / d} e_{a / b}$ or $\beta=$ $h_{c / d} h_{a / b}$, where $(a, b),(c, d)$ are lattice points in the upper half plane with $\operatorname{gcd}(a, b)=\operatorname{gcd}(c, d)=1$ and $a / b<c / d$ and $b+d=Q^{\prime}$. Let

$$
E:=E\left(\frac{a}{b}+\epsilon, \frac{c}{d}-\epsilon ; a+c, b+d\right)
$$

where $0<\epsilon<1 / Q^{2}$, and let $H$ be the sum of all generators obtained by replacing an $e_{p / q}$ factor in $E$ with $h_{p / q}$. In the first two cases for $\beta$ we have $\alpha=E$, while in the third case $\alpha$ is a summand of $H$.

To simplify notation, let $\delta^{*}$ be the dual differential defined by

$$
\left\langle\alpha, \delta^{*} \beta\right\rangle:=\langle\delta \alpha, \beta\rangle \text {. }
$$

Then to prove the lemma it is enough to show that

$$
\begin{gathered}
\delta^{*}\left(e_{c / d} h_{a / b}\right)=\delta^{*}\left(h_{c / d} e_{a / b}\right)=E, \\
\delta^{*}\left(h_{c / d} h_{a / b}\right)=H .
\end{gathered}
$$

We first observe that there can be no other terms in $\delta^{*}\left(e_{c / d} h_{a / b}\right), \delta^{*}\left(h_{c / d} e_{a / b}\right)$, and $\delta^{*}\left(h_{c / d} h_{a / b}\right)$ by Theorem 3.5(a). Now write

$$
E=e_{p_{1} / q_{1}}^{\lambda_{1}} \cdots e_{p_{k} / q_{k}}^{\lambda_{k}}
$$

with $p_{i} / q_{i}>p_{j} / q_{j}$ for $i<j$. We prove equations (31) and (32) in three steps.

Step 1 Suppose $k=1$. Then we show that (31) holds, and if also $\lambda_{1}=1$, or equivalently $b c-a d=1$, then (32) holds.

To do so, we reduce to the paper by Taubes [25] which studies pseudoholomorphic thrice-punctured spheres on $\mathbb{R} \times S^{1} \times S^{2}$ for a certain almost complex structure. By an appropriate identification of $\left[X_{1}, X_{2}\right] \times S^{1}$ with a subset of $S^{2}$ contained between two latitude lines, we deduce from [25, Thm. A.2] that there is an almost complex structure $J_{T}$ on $\mathbb{R} \times Y_{\phi_{0}} \simeq \mathbb{R} \times S^{1} \times\left[X_{1}, X_{2}\right] \times S^{1}$ such that:

(i) $J_{T}$ is $\mathbb{R} \times S^{1} \times S^{1}$ invariant.

(ii) If $s$ denotes the $\mathbb{R}$ coordinate, then $J_{T}$ sends $\partial_{s}$ to a positive multiple of the mapping torus flow $R$. 
(iii) $J_{T}$ is tamed by $\Omega$.

(iv) The moduli space $\mathcal{M}_{T}$ of $J_{T}$-holomorphic thrice-punctured spheres with an outgoing end at $x=(a+c) /(b+d)$ and with incoming ends at $x=a / b$ and $x=c / d$ consists of a single orbit of the $\mathbb{R} \times S^{1} \times S^{1}$ action.

Let $\gamma_{p / q}$ denote the circle of periodic orbits of $\phi_{0}$ at $x=p / q$. Let

$$
\xi: \mathcal{M}_{T} / \mathbb{R} \longrightarrow \gamma_{p_{1} / q_{1}} \times \gamma_{c / d} \times \gamma_{a / b} \simeq\left(S^{1}\right)^{3}
$$

denote the "endpoint map" sending a flow line to the periodic orbits at its ends. The $S^{1}$ actions on $\xi\left(\mathcal{M}_{T} / \mathbb{R}\right)$ by rotation in the $-t$ and $y$ directions have weights $\left(p_{1}, c, a\right)$ and $\left(q_{1}, d, b\right)$ respectively. It follows by (iv) that

$\pm\left[\xi\left(\mathcal{M}_{T} / R\right)\right]=\left(d p_{1}-c q_{1}\right)\left[\gamma_{p_{1} / q_{1}} \gamma_{c / d}\right]+\left(b p_{1}-a q_{1}\right)\left[\gamma_{p_{1} / q_{1}} \gamma_{a / b}\right]+(b c-a d)\left[\gamma_{c / d} \gamma_{a / b}\right]$

in $H_{2}\left(\gamma_{p_{1} / q_{1}} \times \gamma_{c / d} \times \gamma_{a / b}\right)$. Since the triangle with vertices $(0,0),(c, d)$, and $(a+c, b+d)$ contains no lattice points other than the vertices and the $\lambda_{1}-1$ lattice points on the edge in between $(0,0)$ and $(a+c, b+d)$, we get

$$
\pm\left[\xi\left(\mathcal{M}_{T} / R\right)\right]=-\left[\gamma_{p_{1} / q_{1}} \gamma_{c / d}\right]+\left[\gamma_{p_{1} / q_{1}} \gamma_{a / b}\right]+\lambda_{1}\left[\gamma_{c / d} \gamma_{a / b}\right]
$$

We now perturb the setup so that $\gamma_{p / q}$ splits into an elliptic orbit $e_{p / q}$ and a hyperbolic orbit $h_{p / q}$ for $(p, q)=(a, b),(c, d),\left(p_{1}, q_{1}\right)$. By Morse-Bott theory, cf. 1], it follows from (33) that there will be one flow line from $e_{p_{1} / q_{1}}^{\lambda_{1}}$ with one outgoing end to each of $h_{c / d} e_{a / b}$ and $e_{c / d} h_{a / b}$; and when $\lambda_{1}=1$, there will be one flow line from $h_{(a+c) /(b+d)}$ to $h_{c / d} h_{a / b}$. (These flow lines are constructed by a gluing argument, using the fact that $\mathcal{M}_{T}$ is cut out transversely by [25, 26]. There is only one flow line in each case by a compactness argument as in in Appendix A. These flow lines are embedded by the adjunction formula (13).)

To complete the proof of Step 1, we can deform this perturbation of $\left(\phi_{0}, J_{T}\right)$ to $(\phi, J)$ while preserving $\mathbb{R}$-invariance and conditions (ii) and (iii) above. As in Lemma 3.15 the mod 2 count of flow lines as above does not change during the deformation.

Step 2 We now prove equation (31) when $k>1$, assuming it holds whenever the positive integer $b c-a d$ is smaller, using Step 1 and $\left(\delta^{*}\right)^{2}=0$ (which follows from $\delta^{2}=0$ ).

To set up the application of $\left(\delta^{*}\right)^{2}=0$, we need to introduce several vectors in the $(p, q)$-plane. We begin with

$$
(v, w):=\lambda_{k}\left(p_{k}, q_{k}\right)-\left(p_{k-1}, q_{k-1}\right) .
$$


We claim that $(v, w)$ points to the left of $(c, d)$, that is $c w-d v>0$, i.e.

$$
\operatorname{det}\left(\begin{array}{ll}
c & \lambda_{k} p_{k} \\
d & \lambda_{k} q_{k}
\end{array}\right)>\operatorname{det}\left(\begin{array}{cc}
c & p_{k-1} \\
d & q_{k-1}
\end{array}\right) \text {. }
$$

Otherwise the lattice point $(c, d)+(a, b)-2 \lambda_{k}\left(p_{k}, q_{k}\right)$ would lie between $\mathcal{P}(E)$ and $\mathcal{P}\left(e_{c / d} e_{a / b}\right)$ or (if equality holds and $k=2$ and $\lambda_{1}=1$ ) on the line segment between $(0,0)$ and $(c, d)$, contradicting the definition of $E$ or the assumption $\operatorname{gcd}(c, d)=1$. Similarly, $(v, w)$ points to the left of $(a, b)$, as otherwise the lattice point $(c, d)+(a, b)-(v, w)$ would lie between $\mathcal{P}(E)$ and $\mathcal{P}\left(e_{c / d} e_{a / b}\right)$ or on the line segment between $(c, d)$ and $(c, d)+(a, b)$. Hence there exists $A \in \mathrm{SL}_{2} \mathbb{Z}$ sending $(v, w)$ to the upper half plane while keeping $(a, b)$ and $(c, d)$ in the upper half plane. So by Lemma 3.16 we may assume that $w>0$. (By Lemma 3.15] we may assume that $Q$ is large enough that Lemma 3.16 is applicable.)

Consider now the triangle with vertices $(c, d),(c, d)+(a, b)$, and $(c, d)+(a, b)-$ $(v, w)$. Choose a lattice point $(f, g)$ in this triangle with minimal positive distance to the line through $(c, d)$ and $(c, d)+(a, b)$. Define

$$
\begin{aligned}
\left(a^{\prime}, b^{\prime}\right) & :=(f, g)-(c, d), \\
\left(a^{\prime \prime}, b^{\prime \prime}\right) & :=(a, b)-\left(a^{\prime}, b^{\prime}\right), \\
\left(p^{\prime}, q^{\prime}\right) & :=\lambda_{k}\left(p_{k}, q_{k}\right)-\left(a^{\prime \prime}, b^{\prime \prime}\right) .
\end{aligned}
$$

The relevant vectors look something like this:

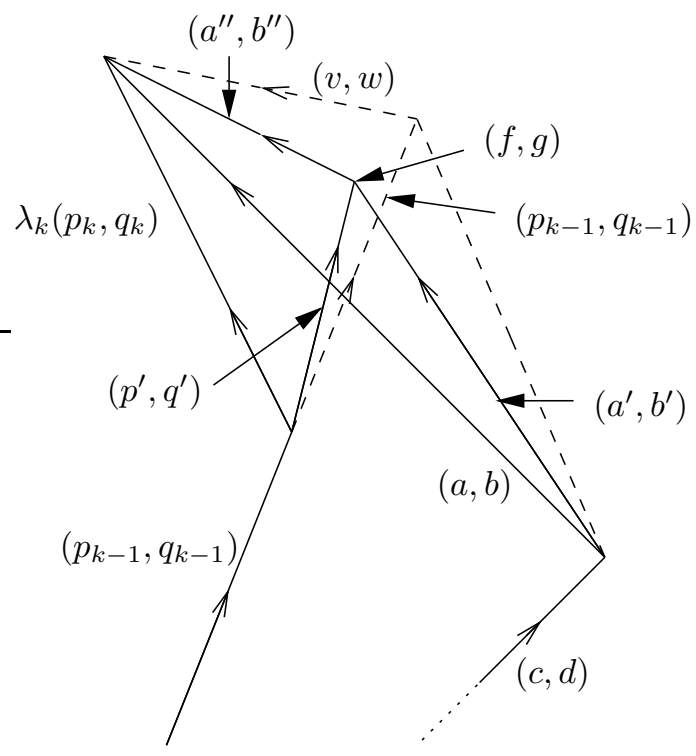


Note that $\left(p^{\prime}, q^{\prime}\right)$ points to the left of or in the same direction as $\left(p_{k-1}, q_{k-1}\right)$, because the triangle with vertices $(c, d),(c, d)+(a, b)-(v, w)$, and $(c, d)+$ $(a, b)-\lambda_{k}\left(p_{k}, q_{k}\right)$ contains no lattice points other than the vertices, because the same is true for the triangle with vertices $(c, d),(c, d)+(a, b)-\lambda_{k}\left(p_{k}, q_{k}\right)$, and $(c, d)+(a, b)-\lambda_{k}\left(p_{k}, q_{k}\right)-\left(p_{k-1}, q_{k-1}\right)$, by definition of $E$.

Also $\left(p^{\prime}, q^{\prime}\right)$ points to the right of $\left(p_{k}, q_{k}\right)$, so $q^{\prime}>0$.

The vector $\left(a^{\prime}, b^{\prime}\right)$ points to the right of $(a, b)$ and to the left of $(c, d)$; in particular $b^{\prime}>0$.

The vector $\left(a^{\prime \prime}, b^{\prime \prime}\right)$ points to the left of $(a, b)$ and to the right of or in the same direction as $(v, w)$; hence $b^{\prime \prime}>0$. Also, since $(a, b)$ and $(v, w)$ both point to the left of $(c, d)$, it follows that $\left(a^{\prime \prime}, b^{\prime \prime}\right)$ does as well; hence

$$
b^{\prime} c-a^{\prime} d<b c-a d .
$$

Now let $E_{0}:=\prod_{i=1}^{k-1} e_{p_{i} / q_{i}}^{\lambda_{i}}$. Since there are no lattice points between the paths $\mathcal{P}(E)$ and $\mathcal{P}\left(e_{c / d} e_{a^{\prime} / b^{\prime}} e_{a^{\prime \prime} / b^{\prime \prime}}\right)$, it follows that

$$
\begin{gathered}
E\left(\frac{a^{\prime}}{b^{\prime}}+\epsilon, \frac{c}{d}-\epsilon ; a^{\prime}+c, b^{\prime}+d\right)=E_{0} e_{p^{\prime} / q^{\prime}}, \\
E\left(\frac{a^{\prime \prime}}{b^{\prime \prime}}+\epsilon, \frac{a^{\prime}}{b^{\prime}}-\epsilon ; a, b\right)=e_{a / b}, \\
E\left(\frac{a^{\prime \prime}}{b^{\prime \prime}}+\epsilon, \frac{p^{\prime}}{q^{\prime}}-\epsilon ; a^{\prime \prime}+p^{\prime}, b^{\prime \prime}+q^{\prime}\right)=e_{p_{k} / q_{k}}^{\lambda_{k}} .
\end{gathered}
$$

By Lemma 3.13, we may assume that $X_{1}$ is small enough so that $a^{\prime \prime} / b^{\prime \prime} \in$ $\left[X_{1}, X_{2}\right]$. Then by inductive hypothesis using (34), and Step 1, we deduce

$$
\begin{aligned}
\delta^{*}\left(e_{c / d} h_{a^{\prime} / b^{\prime}}\right) & =E_{0} e_{p^{\prime} / q^{\prime}}, \\
\delta^{*}\left(h_{a^{\prime} / b^{\prime}} h_{a^{\prime \prime} / b^{\prime \prime}}\right) & =h_{a / b}, \\
\delta^{*}\left(e_{p^{\prime} / q^{\prime}} h_{a^{\prime \prime} / b^{\prime \prime}}\right) & =e_{p_{k} / q_{k}}^{\lambda_{k}} .
\end{aligned}
$$

By equations (35) and (36) and the trivial cylinder lemma 3.9

$$
\delta^{*}\left(e_{c / d} h_{a^{\prime} / b^{\prime}} h_{a^{\prime \prime} / b^{\prime \prime}}\right)=E_{0} e_{p^{\prime} / q^{\prime}} h_{a^{\prime \prime} / b^{\prime \prime}}+e_{c / d} h_{a / b} .
$$

No other terms are possible on the right hand side of (38) by Theorem 3.5(a).

It follows from (37) and the trivial cylinder lemma 3.9 that

$$
\left\langle E, \delta^{*}\left(E_{0} e_{p^{\prime} / q^{\prime}} h_{a^{\prime \prime} / b^{\prime \prime}}\right)\right\rangle=1 .
$$

Since $\left(\delta^{*}\right)^{2}=0$, equations (38) and (39) imply that

$$
\left\langle E, \delta^{*}\left(e_{c / d} h_{a / b}\right)\right\rangle=1
$$


so $\delta^{*}\left(e_{c / d} h_{a / b}\right)=E$.

By a symmetric argument, $\delta^{*}\left(h_{c / d} e_{a / b}\right)=E$.

Step 3 We now prove (32). Suppose first that $k=1$. By Step 1 we may assume that $\lambda_{1}>1$. By (31), Theorem 3.5(a) and the trivial cylinder lemma 3.9 we have

$$
\delta^{*}\left(h_{c / d} e_{a / b} h_{a / b}\right)=e_{p_{1} / q_{1}}^{\lambda_{1}} h_{a / b}+x e_{p_{1} / q_{1}}^{\lambda_{1}-1} h_{p_{1} / q_{1}} e_{a / b},
$$

where $x$ is an unknown coefficient, and

$$
\delta^{*}\left(e_{p_{1} / q_{1}}^{\lambda_{1}} h_{a / b}\right)=\delta^{*}\left(e_{p_{1} / q_{1}}^{\lambda_{1}-1} h_{p_{1} / q_{1}} e_{a / b}\right)=e_{p_{1} / q_{1}}^{\lambda_{1}-1} e_{\left(a+p_{1}\right) /\left(b+q_{1}\right)} .
$$

By $\left(\delta^{*}\right)^{2}=0$ we get $x=1$, and by the trivial cylinder lemma 3.9 and Theorem 3.5. (a) we get $\delta^{*}\left(h_{c / d} h_{a / b}\right)=e_{p_{1} / q_{1}}^{\lambda_{1}-1} h_{p_{1} / q_{1}}$, so (32) holds.

Strictly speaking, equation (40) above makes sense only if $Q$ is sufficiently large, e.g. if $Q \geq 2 Q^{\prime}$. But we can assume this without loss of generality by Lemma 3.15

If $k>1$, we obtain (32) similarly to Step 2, using $\left(\delta^{*}\right)^{2}\left(h_{c / d} h_{a^{\prime} / b^{\prime}} h_{a^{\prime \prime} / b^{\prime \prime}}\right)=0$ and induction on $b c-a d$.

Remark 3.18 If we knew the expected isotopy invariance of $\mathrm{PFH}$, cf. Section 2, then we could give a conceptually simpler proof of steps 1 and 2 above, without using [25], by considering a Dehn twist on a disc. There we can easily calculate the $\mathrm{PFH}$ using isotopy invariance, and together with a spectral sequence similar to the one in Section 3.9, this gives sufficient information about the differential.

Proof of Theorem 3.5(b) This follows immediately from Lemma 3.17 and the trivial cylinder lemma 3.9 .

\subsection{Computing the homology of the cylinder complex}

Proof of Theorem 3.1 We compute the homology combinatorially using Theorem 3.5. We use induction. The base case of the induction is when $P / Q \notin\left[X_{1}, X_{2}\right]$; here the theorem is obvious because the chain complex has no generators. For the inductive step, suppose $P / Q \in\left[X_{1}, X_{2}\right]$. We will prove the theorem assuming that it is true for $\left(X_{1}, X_{2}^{\prime} ; P^{\prime}, Q^{\prime}\right)$ whenever $X_{2}^{\prime}<X_{2}$, $Q^{\prime} \leq Q$, and the interval $\left[X_{1}, X_{2}^{\prime}\right]$ contains fewer rational numbers of denominator $\leq Q$ than the interval $\left[X_{1}, X_{2}\right]$. 
To carry out the inductive step, define a filtration $\mathcal{F}_{0} \supset \mathcal{F}_{-1} \supset \cdots$ of our chain complex as follows. We say that the " $p / q$ exponent" of a generator is the total exponent of $e_{p / q}$ and $h_{p / q}$. Write

$$
E=E\left(X_{1}, X_{2} ; P, Q\right)=e_{p_{1} / q_{1}}^{\lambda_{1}} \cdots e_{p_{k} / q_{k}}^{\lambda_{k}}
$$

with $p_{1} / q_{1}>\cdots>p_{k} / q_{k}$. Let $c=p_{1}$ and $d=q_{1}$. Define $\mathcal{F}_{-i}$ to be the span of all generators with $c / d$ exponent at least $i$. We have $\delta\left(\mathcal{F}_{-i}\right) \subset \mathcal{F}_{-i}$ by Theorem [3.5(a), because if $p / q>c / d$, then no generators have positive $p / q$ exponent; so rounding a corner or double rounding never increases the $c / d$ exponent.

From this filtered complex we obtain a spectral sequence $\mathcal{E}_{*, *}^{*}$ which converges to $H P_{*}\left(X_{1}, X_{2} ; P, Q\right)$. The $\mathcal{E}^{1}$ term is the homology of the associated graded complex $\mathcal{G}_{-i}=\mathcal{F}_{-i} / \mathcal{F}_{-i-1}$. By Theorem 3.5(a), if $\alpha$ and $\beta$ are generators with the same $c / d$ exponent, then $\langle\delta \alpha, \beta\rangle=1$ only if $\alpha$ is obtained from $\beta$ by rounding a corner or double rounding not involving $c / d$, so

$$
\begin{aligned}
H_{*}\left(\mathcal{G}_{0}\right) & =H P_{*}\left(X_{1}, c / d-\epsilon ; P, Q\right), \\
H_{*}\left(\mathcal{G}_{-i}\right) & =\operatorname{span}\left\{e_{c / d}^{i}, e_{c / d}^{i-1} h_{c / d}\right\} \otimes H P_{*}\left(X_{1}, \frac{c}{d}-\epsilon ; P-i c, Q-i d\right), \quad i>0 .
\end{aligned}
$$

We now use the inductive hypothesis to make this more explicit. Let $l=$ $\lambda_{1}$ above. Then $H_{*}\left(\mathcal{G}_{-i}\right)=0$ for $i>l$, while for $i \leq l$, the homology $H P_{*}\left(X_{1}, c / d-\epsilon ; P-i c, Q-i d\right)$ is generated by the homology class of

$$
E_{i}:=E\left(X_{1}, \frac{c}{d}-\epsilon ; P-i c, Q-i d\right),
$$

together with the homology class $\left[H_{i}\right]$, where $H_{i}$ denotes a generator obtained by replacing one of the $e_{p / q}$ factors in $E_{i}$ by $h_{p / q}$.

We now relate $E_{i}$ to $E_{i-1}$. Suppose $0<i \leq l$, and write

$$
E_{i}=e_{p_{1} / q_{1}}^{\lambda_{1}} \cdots e_{p_{k} / q_{k}}^{\lambda_{k}}=: e_{p_{1} / q_{1}} E_{i}^{\prime} .
$$

with $p_{1} / q_{1}>\cdots>p_{k} / q_{k}$. (Here we are recycling the notation so that $p_{j}, q_{j}$, $\lambda_{j}, k$ are different from (41).) Now $\left(p_{1}, q_{1}\right)$ is on the boundary of the convex hull of the set of lattice points in the parallelogram $Z\left(X_{1}, c / d-\epsilon ; P-i c, Q-i d\right)$. It follows that $\left(p_{1}+c, q_{1}+d\right)$ is on the boundary of the convex hull of the set of lattice points in $Z\left(X_{1}, c / d-\epsilon ; P-(i-1) c, Q-(i-1) d\right)$. For example, the case $k=4$ and $\lambda_{1}=1$ looks like this: 


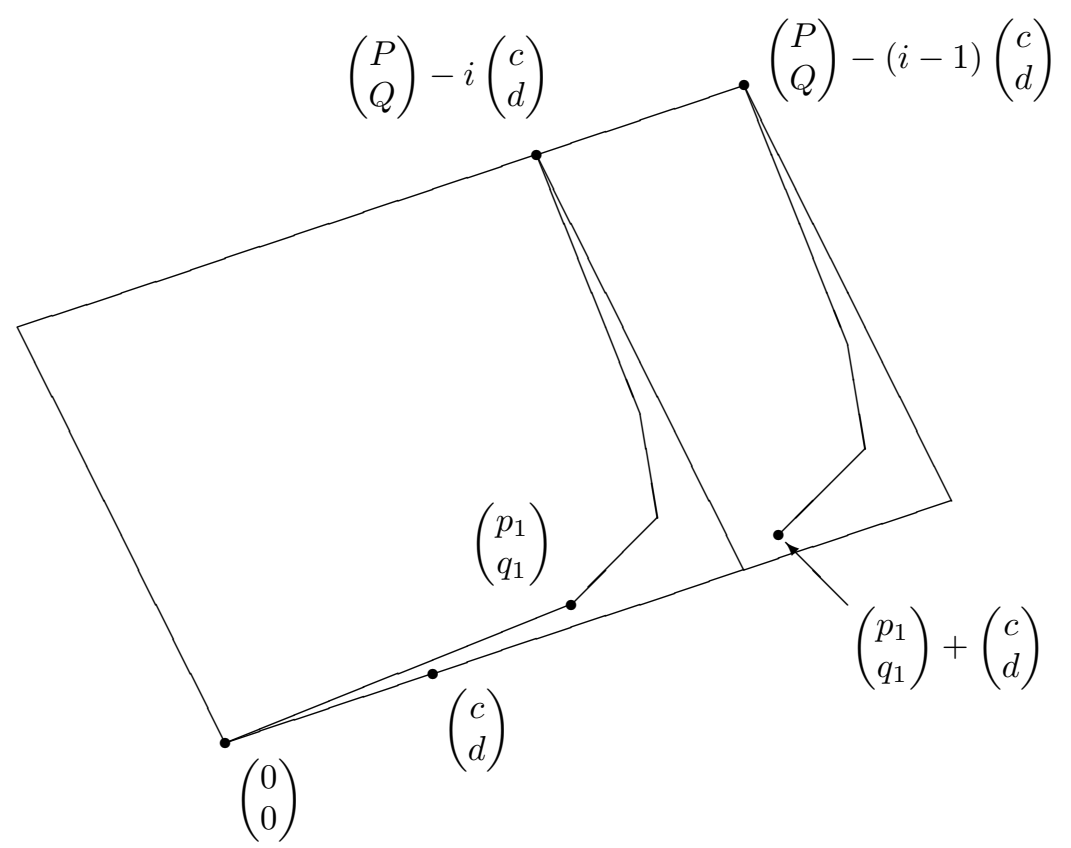

We deduce that

$$
E_{i-1}=E\left(\frac{p_{1}}{q_{1}}+\epsilon, \frac{c}{d}-\epsilon ; p_{1}+c, q_{1}+d\right) E_{i}^{\prime} .
$$

By Theorem 3.5(b), it follows that if we choose $H_{i}=h_{p_{1} / q_{1}} E_{i}^{\prime}$, then

$$
\begin{aligned}
\left\langle\delta\left(e_{c / d}^{i-1} E_{i-1}\right), e_{c / d}^{i-1} h_{c / d} E_{i}\right\rangle=\left\langle\delta\left(e_{c / d}^{i-1} E_{i-1}\right), e_{c / d}^{i} H_{i}\right\rangle & =1, \\
\left\langle\delta\left(e_{c / d}^{i-2} h_{c / d} E_{i-1}\right), e_{c / d}^{i-1} h_{c / d} H_{i}\right\rangle & =1 .
\end{aligned}
$$

Also, if we choose a representative $H_{i-1}$ of the class $\left[H_{i-1}\right]$ by replacing one of the $e_{p / q}$ factors in $E\left(p_{1} / q_{1}+\epsilon, c / d-\epsilon ; p_{1}+c, q_{1}+d\right)$ by $h_{p / q}$, then

$$
\left\langle\delta\left(e_{c / d}^{i-1} H_{i-1}\right), e_{c / d}^{i-1} h_{c / d} H_{i}\right\rangle=1 .
$$

Finally, by Theorem 3.5(a), replacing $H_{i}$ with another generator would change the corresponding differential coefficients above to zero.

It follows that the first differential in the spectral sequence, which we denote by $\partial_{1}$, satisfies

$$
\begin{aligned}
\partial_{1}\left(e_{c / d}^{i-1}\left[E_{i-1}\right]\right) & =e_{c / d}^{i-1} h_{c / d}\left[E_{i}\right]+e_{c / d}^{i}\left[H_{i}\right], \\
\partial_{1}\left(e_{c / d}^{i-2} h_{c / d}\left[E_{i-1}\right]\right) & =\partial_{1}\left(e_{c / d}^{i-1}\left[H_{i-1}\right]\right)=e_{c / d}^{i-1} h_{c / d}\left[H_{i}\right] .
\end{aligned}
$$


The differential $\partial_{1}$ cannot contain any other terms due to the bigrading, as we see by laying out the $\mathcal{E}^{1}$ term:

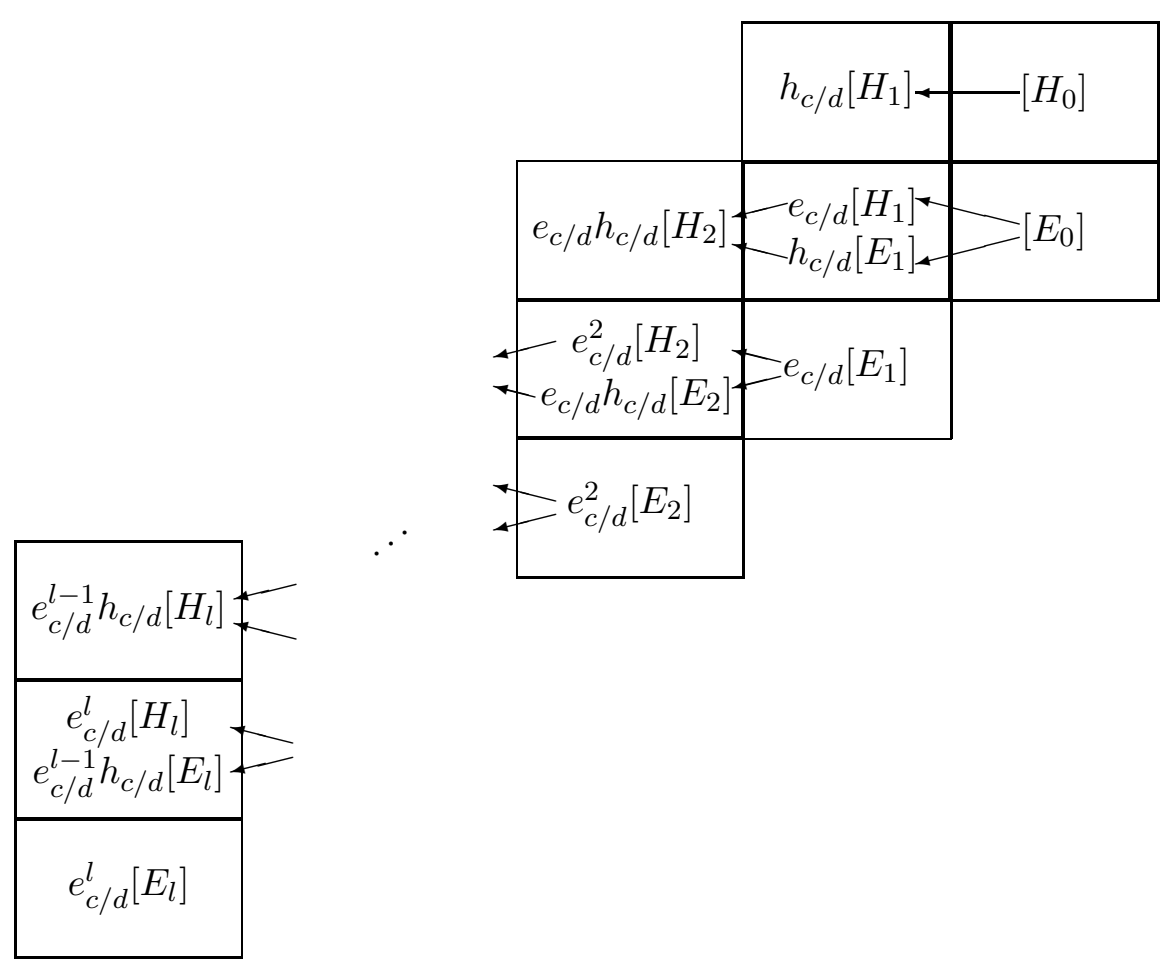

From this picture we see that everything is killed in the spectral sequence, except for

$$
e_{c / d}^{l}\left[E_{l}\right]=\left[E\left(X_{1}, X_{2} ; P, Q\right)\right],
$$

and two generators which become homologous in $\mathcal{E}^{2}$, namely

$$
e_{c / d}^{l}\left[H_{l}\right], \quad e_{c / d}^{l-1} h_{c / d}\left[E_{l}\right]
$$

Thus $H P_{*}\left(X_{1}, X_{2} ; P, Q\right)$ is exactly as described in Theorem 3.1

Remark 3.19 The above algebraic calculation can be simplified after introducing some more general combinatorial chain complexes involving rounding corners of polygonal paths. Compare [11, Prop. 5.5]. 


\section{PFH of a Dehn twist on a torus}

Let $n$ be a positive integer. We now consider the composition $\phi_{0}^{T}$ of $n$ parallel positive Dehn twists on the torus from equation (3). We can identify

$$
H_{1}\left(Y_{\phi_{0}^{T}}\right) \simeq \mathbb{Z} \oplus \mathbb{Z} \oplus \mathbb{Z} / n
$$

such that the circle of periodic orbits at $x=p / q$ is sent to $(q, 0,-p \bmod n)$. Thus for each degree $d>0$, there are $n$ sectors $h \in H_{1}(Y)$ containing homology classes of orbit sets, classified by the $\bmod n$ total numerator $[P] \in \mathbb{Z} / n$. In this section we compute $H P_{*}\left(\phi^{T}, h\right)$, where $\phi^{T}$ is a modification of $\phi_{0}^{T}$ as in Section 3.1 with $Q=d$, and $J$ is any generic almost complex structure such that $\left(\phi^{T}, J\right)$ is $d$-regular. We denote this $\mathrm{PFH}$ by $H P_{*}\left(\phi^{T} ;[P], d\right)$; this is noncanonically $\mathbb{Z} / 2 d$-graded, see Section 2. Since the isotopy from $\phi_{0}^{T}$ to $\phi^{T}$ is Hamiltonian, $\left(\phi^{T}, h\right)$ is monotone, see Lemma 5.1

Theorem 4.1 For every $[P] \in \mathbb{Z} / n$ and $d \in \mathbb{Z}^{>0}$, the periodic Floer homology

$$
H P_{i}\left(\phi^{T} ;[P], d\right) \simeq \mathbb{Z} / 2
$$

for each value of the $\mathbb{Z} / 2 d$-grading $i$.

In the proof we use the following notation. As with the cylinder, for $0<p / q<n$ and $q \leq d$ there are periodic orbits $e_{p / q}$ and $h_{p / q}$ at $x=p / q$ of period $q$. We denote the two fixed points at $x=0 \bmod n$ simply by $e$ and $h$. By symmetry, we may assume without loss of generality that $P \equiv 0 \bmod n$.

\subsection{The wrapping spectral sequence}

The map $\phi_{0}^{T}$ has a circle of fixed points at $x \equiv 0 \bmod n$, each of which corresponds to a circle in $Y_{\phi_{0}^{T}}$. The isotopy from $\phi_{0}^{T}$ to $\phi^{T}$ induces a piecewise smooth homeomorphism $Y_{\phi_{0}^{T}} \rightarrow Y_{\phi^{T}}$. Let $\rho \subset Y_{\phi^{T}}$ be the image of one of the circles in $Y_{\phi_{0}^{T}}$ coming from a fixed point which does not survive under the perturbation.

Definition 4.2 If $C$ is a flow line for $\left(\phi^{T}, J\right)$, define the wrapping number

$$
\eta(C):=\#(C \cap(\mathbb{R} \times \rho)) \in \mathbb{Z} .
$$


Note that $\eta(C)$ depends only on the relative homology class of $C$, and does not depend on $\rho$ since all such circles are homotopic in the complement of the set of periodic orbits of period $\leq d$. We remark that $\eta$ is reminiscent of the quantity $n_{z}$ considered in [17.

Choose $0<\epsilon<1 / d$ so that $\phi^{T}$ agrees with $\phi_{0}^{T}$ near $x= \pm \epsilon \bmod n$. If $\alpha$ is an orbit set, let $d_{0}(\alpha)$ denote the total exponent of $e$ and $h$ in $\alpha$.

Lemma 4.3 If $C \in \mathcal{M}(\alpha, \beta)$, then $\eta(C) \geq 0$. If $\eta(C)=0$, then:

$$
d_{0}(\beta)-d_{0}(\alpha) \geq\left\{\begin{array}{ll}
1 & \text { if } C \cap\{x=\epsilon\} \neq \emptyset \\
0 & \text { otherwise }
\end{array}+ \begin{cases}1 & \text { if } C \cap\{x=-\epsilon\} \neq \emptyset \\
0 & \text { otherwise. }\end{cases}\right.
$$

Proof Perturb $\epsilon$ so that $C$ is transverse to $\{x= \pm \epsilon\}$. Let

$$
\left(p_{ \pm}, q_{ \pm}\right):=[C \cap\{x= \pm \epsilon\}] \in H_{1}\left(S_{y}^{1} \times S_{t}^{1}\right),
$$

with the sign conventions of Section 3.4. Evidently

$$
\begin{aligned}
p_{ \pm} & =\eta(C), \\
q_{+}-q_{-} & =d_{0}(\beta)-d_{0}(\alpha) .
\end{aligned}
$$

By Lemma 3.11 we have $p_{ \pm} \pm \epsilon q_{ \pm} \geq 0$ with equality only if $C \cap\{x= \pm \epsilon\}=\emptyset$. The lemma follows.

Since all flow lines have nonnegative wrapping number, we can write

$$
\delta=\delta_{0}+\delta_{1}+\delta_{2}+\cdots
$$

where $\delta_{i}$ counts the contributions from flow lines $C$ with $\eta(C)=i$. Since $\delta^{2}=0$ and $\eta$ is additive under gluing of flow lines, it follows that $\delta_{0}^{2}=0$, $\delta_{0} \delta_{1}=\delta_{1} \delta_{0}$, and so forth.

By the index ambiguity formula (7), if $C \in \mathcal{M}(\alpha, \beta)$, we can write

$$
I(C)=I_{0}(\alpha, \beta)+2 d \cdot \eta(C) .
$$

In other words, our chain module has a relative $\mathbb{Z}$-grading $I_{0}$, and $\delta_{\eta}$ shifts this grading by $2 d \eta-1$. We choose an absolute $\mathbb{Z}$-grading by declaring the index of $e^{d}$ to be 0 . In particular, we have a $\mathbb{Z}$-graded $\delta_{0}$-homology $H_{*}\left(\delta_{0}\right)$, and $\delta_{1}$ induces a map $\left(\delta_{1}\right)_{*}$ on it of degree $2 d-1$.

By applying the following general algebraic lemma we obtain a spectral sequence whose $\mathcal{E}^{1}$ term is $H_{*}\left(\delta_{0}\right)$, whose first differential is $\left(\delta_{1}\right)_{*}$, and which converges to the $\mathrm{PFH}$. We call this the wrapping spectral sequence. 
Lemma 4.4 Let $C_{*}$ be a bounded $\mathbb{Z}$-graded vector space, and let

$$
\delta=\delta_{0}+\delta_{1}+\delta_{2}+\cdots: C_{*} \rightarrow C_{*}
$$

satisfy $\delta^{2}=0$ and $\operatorname{deg}\left(\delta_{i}\right)=N i-1$ with $N \neq 0$. Then there is a spectral sequence $\left(\mathcal{E}^{*}, \hat{\delta}_{*}\right)$ such that:

- $\mathcal{E}^{r}$ is $\mathbb{Z}$-graded and $\operatorname{deg}\left(\hat{\delta}_{r}\right)=N r-1$.

- $\mathcal{E}_{*}^{1}=H_{*}\left(\delta_{0}\right)$, and $\hat{\delta}_{1}=\left(\delta_{1}\right)_{*}: H_{*}\left(\delta_{0}\right) \rightarrow H_{*+N-1}\left(\delta_{0}\right)$.

- For $m \in \mathbb{Z} / N$ we have $H_{m}(\delta) \simeq \bigoplus_{i \equiv m \bmod N} \mathcal{E}_{i}^{\infty}$.

Proof If $k$ is an integer, let $C_{*}[k]$ denote $C_{*}$ with the grading shifted by $k$. Define a complex $\widetilde{C}_{*}:=\bigoplus_{i \in \mathbb{Z}} C_{*}[N i]$, with a filtration

$$
\mathcal{F}_{i} \widetilde{C}_{*}:=\bigoplus_{j \leq i} C_{*}[N j]
$$

where the component of the differential from $C_{*}[N i]$ to $C_{*-1}[N j]$ is induced by $\delta_{i-j}$. The filtration gives rise to a spectral sequence $\left(\widetilde{\mathcal{E}}_{*, *}^{*}, \widetilde{\delta}_{*}\right)$ with $\widetilde{\mathcal{E}}_{p, q}^{r} \simeq$ $\widetilde{\mathcal{E}}_{p-1, q-N+1}^{r}$. We then take $\mathcal{E}_{q}^{r}:=\widetilde{\mathcal{E}}_{0, q}^{r}$, with $\hat{\delta}_{r}$ induced from $\widetilde{\delta}_{r}$. Clearly $\left(\mathcal{E}^{*}, \hat{\delta}_{*}\right)$ satisfies the first two properties; since $C_{*}$ is bounded and $N \neq 0$, this spectral sequence converges, giving the third property.

\subsection{Lifting from the torus to the cylinder}

To compute the differentials $\delta_{\eta}$, we need to relate flow lines for a Dehn twist on a torus to flow lines for a Dehn twist on a cylinder. Under the covering

$$
\mathbb{R} \times S^{1} \longrightarrow(\mathbb{R} / n \mathbb{Z}) \times S^{1}
$$

of the torus by the infinite cylinder, the symplectomorphism $\phi^{T}$ of $(\mathbb{R} / n \mathbb{Z}) \times S^{1}$ lifts to a perturbation $\phi$ of the cylinder twist $\phi_{0}$ on $\mathbb{R} \times S^{1}$. There is then a covering of mapping tori

$$
\pi: \mathbb{R} \times Y_{\phi} \longrightarrow \mathbb{R} \times Y_{\phi^{T}},
$$

and the almost complex structure $J$ for $\phi^{T}$ pulls back to an almost complex structure $\pi^{*} J$ for $\phi$, such that $\left(\phi, \pi^{*} J\right)$ satisfy the conditions in Section 3.1

The following lemma shows that to compute the $\mathrm{PFH}$ of $\left(\phi^{T}, J\right)$ on the torus, we need only consider flow lines that lift to flow lines for $\left(\phi, \pi^{*} J\right)$ on the cylinder. (This lemma is actually used only for a small part of the calculation in Section 4.4.) 
Lemma 4.5 Let $\delta^{\prime}$ denote the contribution to the PFH differential for the torus coming from flow lines that do not lift via $\pi$ to flow lines for the cylinder. Then $\delta^{\prime}=0$.

Proof Let $C$ be an $I=1$ flow line for the torus. As in Section 3.2 since $I(C)=1$ we can write $C=C^{\prime} \cup T$ where $C^{\prime}$ is nontrivial and connected and $T$ is a union of trivial cylinders. As in equation (26) we have

$$
2 g\left(C^{\prime}\right)+2 e_{-}\left(C^{\prime}\right)+h\left(C^{\prime}\right)=3 .
$$

In particular $g\left(C^{\prime}\right) \in\{0,1\}$. Now $C$ lifts to the cylinder if and only if $g\left(C^{\prime}\right)=1$. For if $g\left(C^{\prime}\right)=0$, then $C^{\prime}$ lifts to the cylinder since each periodic orbit lifts. Conversely, if $g\left(C^{\prime}\right)=1$, then equation (46) implies that $C^{\prime}$ is a flow line from $\alpha$ to $\beta$, where $\alpha$ contains only elliptic factors and $\beta=h_{p / q}$ for some $p, q$. Such a $C^{\prime}$ cannot lift to the cylinder, since a lift would have $I<0$ by Proposition 3.2 and hence does not exist for generic $J$.

By Lemma A.1 for a certain (not locally linear) pair $\left(\phi^{\prime}, J^{\prime}\right)$ where $\phi^{\prime}$ is close to $\phi_{0}^{T}$, there are no genus 1 flow lines from $\alpha$ to $\beta$ as above. Similarly to Lemma 3.15, during a generic deformation of $\left(\phi^{\prime}, J^{\prime}\right)$ to $\left(\phi^{T}, J\right)$, there are never any genus 1 broken GFL's from $\alpha$ to $\beta$ respecting the outgoing partitions at $\alpha$, so the mod 2 count of $I=1$ genus 1 flow lines from $\alpha$ to $\beta$ remains zero.

Going in the other direction, if $C$ is a flow line for the cylinder, then it projects to a generalized flow line $\pi(C)$ for the torus.

Lemma 4.6 If $C$ is a flow line for the cylinder and if $I(\pi(C))=1$, then $\pi(C)$ is a flow line for the torus.

Proof This follows immediately from Corollary 2.2

We now compute the wrapping number $\eta(\pi(C))$. If $\alpha=\gamma_{1} \cdots \gamma_{k}$ is an orbit set for the cylinder where $\gamma_{i}=e_{p_{i} / q_{i}}$ or $\gamma_{i}=h_{p_{i} / q_{i}}$, define

$$
\widetilde{\eta}(\alpha):=\sum_{i=1}^{k}\left\lfloor\frac{p_{i}}{n q_{i}}\right\rfloor\left(-p_{i}+\frac{n q_{i}}{2}\left(\left\lfloor\frac{p_{i}}{n q_{i}}\right\rfloor+1\right)\right) .
$$

Lemma 4.7 Let $C \in \mathcal{M}(\alpha, \beta)$ be a flow line for the cylinder. Then its projection to the torus has wrapping number

$$
\eta(\pi(C))=\widetilde{\eta}(\alpha)-\widetilde{\eta}(\beta) .
$$


Proof By equations (43) and (28), using the notation $p_{i}, q_{i}, p_{j}^{\prime}, q_{j}^{\prime}$, and $q_{-}$ from those equations, we have

$$
\begin{aligned}
\left(\eta(\pi(C)), q_{-}\right) & =[\pi(C) \cap\{x=-\epsilon\}] \\
& =\sum_{m \in \mathbb{Z}} \pi_{*}[C \cap\{x=m n-\epsilon\}] \\
& =\sum_{m \in \mathbb{Z}} \pi_{*}\left(\sum_{p_{i} / q_{i}>m n-\epsilon}\left(-p_{i}, q_{i}\right)-\sum_{p_{j}^{\prime} / q_{j}^{\prime}>m n-\epsilon}\left(-p_{j}^{\prime}, q_{j}^{\prime}\right)\right) \\
& =\sum_{m \in \mathbb{Z}}\left(\sum_{p_{i} / q_{i} \geq m n}\left(-p_{i}+m n q_{i}, q_{i}\right)-\sum_{p_{j}^{\prime} / q_{j}^{\prime} \geq m n}\left(-p_{j}^{\prime}+m n q_{j}^{\prime}, q_{j}^{\prime}\right)\right) .
\end{aligned}
$$

Note that the sum over $m$ has only finitely many nonzero terms. The lemma follows by a straightforward evaluation of this sum.

The following lemma makes it easy to compute the differential $\delta_{0}$ in the wrapping spectral sequence.

Lemma 4.8 Lifting and projecting give a correspondence

$$
\{I=1, \eta=0 \text { torus flow lines }\} \longleftrightarrow\left\{I=1 \text { flow lines for }(-\epsilon, n+\epsilon) \times S^{1}\right\} \text {. }
$$

This is a bijection, except that components of flow lines for the torus in a neighborhood of $\{x=0\}$ can be lifted in two ways, to a neighborhood of $\{x=0\}$ or to a neighborhood of $\{x=n\}$.

Proof $(\rightarrow)$ Let $C$ be a flow line for the torus with $I(C)=1$ and $\eta(C)=0$. We need to show that $C$ lifts to an $I=1$ GFL for $(-\epsilon, n+\epsilon) \times S^{1}$. Clearly any GFL lifting $C$ is a flow line, and unique modulo the proviso in the lemma. Write $C=C^{\prime} \cup T$ where $C^{\prime}$ is a nontrivial, connected flow line from $\alpha$ to $\beta$, and $T$ is a union of trivial cylinders.

We first show that $C^{\prime}$ lifts to a flow line for $(-\epsilon, n+\epsilon) \times S^{1}$. If $C^{\prime}$ does not intersect both of $\{x=\epsilon \bmod n\}$ and $\{x=-\epsilon \bmod n\}$, then $C^{\prime}$ trivially lifts to $(-\epsilon, n+\epsilon) \times S^{1}$. If $C^{\prime}$ intersects both these regions, then Lemma 4.3 implies that $d_{0}(\beta) \geq 2$. As in Lemma 3.8 (c), any incoming elliptic end of $C^{\prime}$ has multiplicity one, so it follows from equation (46) that $\beta=e h$ and $g\left(C^{\prime}\right)=0$, so $C^{\prime}$ lifts to $\mathbb{R} \times S^{1}$. After translation we can choose the lift of $C^{\prime}$ so that $\beta$ is lifted to $h_{n k} e_{0}$ or $e_{n k} h_{0}$ with $k$ a positive integer. By Propositions 3.2 and 3.12 $\alpha$ is lifted to $e_{1 / 2}$ if $n k=1$, and $e_{n k-1} e_{1}$ otherwise. Lemma 4.7] implies 
that $\eta(C)=\eta\left(C^{\prime}\right)=k-1$. Therefore $k=1$. By Lemma 3.11, the lift is supported over $(-\epsilon, n+\epsilon) \times S^{1}$.

We can also lift $T$ to obtain a lift of $C$. The calculations in the proof of Lemma 3.7 show that the lifted flow line $\widetilde{C}$ satisfies $Q_{\tau}([\widetilde{C}])=Q_{\tau}([C])$. Together with equations (20), (21) and (22), this implies that $I(\widetilde{C})=I(C)$.

$(\leftarrow)$ Let $C$ be an $I=1$ flow line for $(-\epsilon, n+\epsilon) \times S^{1}$; we need to show that the projection $\pi(C)$ is a flow line for the torus with $I=1$ and $\eta=0$. As above, $I(\pi(C))=I(C)$. By Lemma 4.6, $\pi(C)$ is a flow line. Lemma 4.7 implies that $\eta(\pi(C))=0$, because if $\alpha$ is an orbit set for $(-\epsilon, n+\epsilon) \times S^{1}$, then $\widetilde{\eta}(\alpha)=0$ by equation (47) since $0 \leq p_{i} / n q_{i} \leq 1$ for each $i$.

\subsection{The $\eta=0$ homology}

We now compute the first term of the wrapping spectral sequence from Section 4.1

Lemma 4.9 For $d>0$ and $P \equiv 0 \bmod n$, the $\eta=0$ homology of a Dehn twist on a torus is given by

$$
H_{*}\left(\delta_{0}\right)=\left\{\begin{array}{cl}
\mathbb{Z} / 2 & \text { if } 0 \leq * \leq 2 d-1 \\
0 & \text { otherwise }
\end{array}\right.
$$

Proof We define a filtration on our chain complex by setting $\mathcal{F}_{i}$ to be the span of those generators in which the total exponent of $e$ and $h$ is at least $d-i$. By Lemma 4.3 we have $\delta_{0}\left(\mathcal{F}_{i}\right) \subset \mathcal{F}_{i}$. Hence we obtain a spectral sequence which converges to the $\eta=0$ homology.

The $\mathcal{E}^{1}$ term is given as follows. Lemma 4.8 implies that the homology of the associated graded complex is given in terms of the cylinder complex by

$$
\begin{aligned}
& H_{*}\left(\mathcal{G}_{0}\right)=\operatorname{span}\left\{e^{d}, e^{d-1} h\right\}, \\
& H_{*}\left(\mathcal{G}_{i}\right)=\operatorname{span}\left\{e^{d-i}, e^{d-i-1} h\right\} \otimes \bigoplus_{k=1}^{i-1} H P_{*}(\epsilon, n-\epsilon ; n k, i), \quad 2 \leq i \leq d-1, \\
& H_{*}\left(\mathcal{G}_{d}\right)=\bigoplus_{k=1}^{d-1} H P_{*}(\epsilon, n-\epsilon ; n k, d),
\end{aligned}
$$

and $H_{*}\left(\mathcal{G}_{i}\right)=0$ for all other $i$. By Theorem 3.1 if $0<k<i$, then the homology $H P_{*}(\epsilon, n-\epsilon ; n k, i)$ is generated by the class

$$
E_{k, i}:=[E(\epsilon, n-\epsilon ; n k, i)],
$$


together with the homology class $H_{k, i}$ of those generators obtained from the generator $E(\epsilon, n-\epsilon ; n k, i)$ by replacing an $e_{p / q}$ factor with $h_{p / q}$.

We now compute the first differential of the spectral sequence, which we denote here by $\partial_{1}$. By Lemma 4.8 all contributions to $\partial_{1}$ come from projections to the torus of differential coefficients in the cylinder complex $C P_{*}(-\epsilon, n+\epsilon ; *, d)$ which increase the total of the 0 -exponent and the $n$-exponent by 1 . When the $n$-exponent increases by one, the differential coefficients are described by (42) with $X_{1}=\epsilon, X_{2}=n+\epsilon, c / d=n / 1$. When the 0 -exponent increases by one the differential coefficients have a similar form by symmetry. We conclude that

$$
\begin{aligned}
\partial_{1}\left(e^{d-i} E_{k, i}\right)= & e^{d-i} h\left(E_{k, i-1}+E_{k-1, i-1}\right) \\
& +e^{d-i+1}\left(H_{k, i-1}+H_{k-1, i-1}\right), \\
\partial_{1}\left(e^{d-i-1} h E_{k, i}\right)= & \partial_{1}\left(e^{d-i} H_{k, i}\right) \\
= & e^{d-i} h\left(H_{k, i-1}+H_{k-1, i-1}\right), \\
\partial_{1}\left(e^{d-i-1} h H_{k, i}\right)= & 0 .
\end{aligned}
$$

\begin{tabular}{|c|c|c|c|}
\hline & & $\begin{array}{l}h\left[e_{1} h_{3 / 2}=h_{1} e_{3 / 2}\right] \\
h\left[e_{1 / 2} h_{1}=h_{1 / 2} e_{1}\right]\end{array}$ & $\begin{array}{c}{\left[e_{3 / 2} h_{3 / 2}\right]} \\
=\left[e_{1 / 2} h_{3 / 2}=h_{1 / 2} e_{3 / 2}\right] \\
{\left[e_{1 / 2} h_{1 / 2}\right]}\end{array}$ \\
\hline & $e h\left[e_{1} h_{1}\right.$ & $\begin{array}{c}e\left[e_{1} h_{3 / 2}=h_{1} e_{3 / 2}\right] \\
h\left[e_{1} e_{3 / 2}\right] \\
e\left[e_{1 / 2} h_{1}=h_{1 / 2} e_{1}\right] \\
h\left[e_{1 / 2} e_{1}\right]\end{array}$ & $\begin{array}{l}{\left[e_{3 / 2}^{2}\right]} \\
=\left[e_{1 / 2} e_{3 / 2}\right] \\
=\left[e_{1 / 2}^{2}\right]\end{array}$ \\
\hline$e^{3} h$ & $\begin{array}{c}e^{2}\left[e_{1} h_{1}\right] \\
e h\left[e_{1}^{2}\right]\end{array}$ & 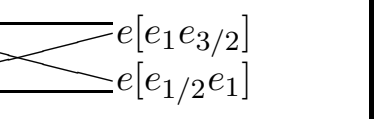 & \\
\hline$e^{4}$ & $e^{2}\left[e_{1}^{2}\right]$ & & \\
\hline
\end{tabular}

In this equation, when $k=1$ we interpret $E_{k-1, i-1}:=H_{k-1, i-1}:=0$, and when $k=i-1$ we interpret $E_{k, i-1}:=H_{k, i-1}:=0$.

For example, if $n=2$ and $d=4$, then $\left(\mathcal{E}^{1}, \partial_{1}\right)$ is as follows:

Here the notation $[a=b]$ means that one can choose $a$ or $b$, and one will obtain the same homology class either way. 
Equation (48) implies that the $\mathcal{E}^{2}$ term is given by

$$
\begin{aligned}
\mathcal{E}_{0,0}^{2} & =\operatorname{span}\left\{e^{d}\right\}, \\
\mathcal{E}_{0,1}^{2} & =\operatorname{span}\left\{e^{d-1} h\right\}, \\
\mathcal{E}_{i, i-2}^{2} & =\operatorname{span}\left\{e^{d-i} \sum_{k=1}^{i-1} E_{k, i}\right\}, \quad 2 \leq i \leq d, \\
\mathcal{E}_{i, i-1}^{2} & =\operatorname{span}\left\{\sum_{k=1}^{i-1} e^{d-i-1}\left[h E_{k, i}=e H_{k, i}\right]\right\}, \quad 2 \leq i \leq d-1, \\
\mathcal{E}_{d, d-1}^{2} & =\operatorname{span}\left\{\sum_{k=1}^{d-1} H_{k, d}\right\},
\end{aligned}
$$

and all other $\mathcal{E}_{i, j}^{2}$ 's are zero. In equation (49), strictly speaking there should be square brackets around each generator to indicate that it is a homology class in $\mathcal{E}^{2}$. Here we have inferred the bigrading from the various nonvanishing differential coefficients; this can also be computed directly.

Equation (49) shows that $\mathcal{E}^{2}$ has one generator in each degree from 0 to $2 d-1$; so to complete the proof, we must show that our spectral sequence degenerates at $\mathcal{E}^{2}$. Because of the bigrading, the only possible nonzero higher differential coefficient is $\left\langle\partial_{2}\left(e^{d-2} E_{1,2}\right), e^{d-1} h\right\rangle$, which by the trivial cylinder lemma 3.9 equals the $d=2$ differential coefficient $\left\langle\partial_{2}\left(E_{1,2}\right)\right.$,eh $\rangle$. But this differential coefficient vanishes, because in the cylinder complex $C P_{*}(-\epsilon, n+\epsilon ; n, 2)$ we have $\delta\left(E_{1,2}\right)=e_{n} h_{0}+h_{n} e_{0}$, which in the torus complex projects to $e h+h e=0$.

\subsection{Degeneration of the wrapping spectral sequence}

We will now see that although there do exist index 1 flow lines with $\eta>0$, these do not contribute in the wrapping spectral sequence.

Proof of Theorem 4.1 We compute $H_{*}(\delta)$ using Lemma 4.4. Lemma 4.9 tells us that $H_{*}\left(\delta_{0}\right)$ has one generator in each index $0, \ldots, 2 d-1$, and we saw in the proof that $\left[e^{d}\right]$ is the generator of index 0 . Since $\delta_{\eta}$ shifts the grading by $2 d \eta-1$, it will suffice to show that

$$
\left(\delta_{1}\right)_{*}\left[e^{d}\right]=0 \in H_{*}\left(\delta_{0}\right) .
$$

By Lemma 4.5. to compute $\delta_{1}$, it suffices to consider flow lines that lift to the cylinder $\mathbb{R} \times S^{1}$. As in Lemma 3.8 (c), the nontrivial component of $C$ has only 
one outgoing end at $e$, so we can choose the lifted flow line $C$ to be from $e_{0}^{d}$ to some $\beta$.

If $C$ is an $I=1$ flow line for the cylinder from $e_{0}^{d}$ to $\beta$, then by Lemma 4.6. $\pi(C)$ contributes to $\delta_{1}\left(e^{d}\right)$ if and only if $\eta(\pi(C))=I(\pi(C))=1$. By equation (45) and Lemma 4.7. this holds if and only if $\widetilde{\eta}(\beta)=-1$ and $I_{0}\left(e^{d}, \pi(\beta)\right)=1-2 d$. Therefore

$$
\delta_{1}\left(e^{d}\right)=\sum_{-\widetilde{\eta}(\beta)=I_{0}\left(e^{d}, \pi(\beta)\right)+2 d=1}\left\langle\delta e_{0}^{d}, \beta\right\rangle \pi(\beta)
$$

where $\beta$ is a generator for the cylinder and $\pi(\beta)$ denotes its projection to the torus.

In the cylinder, by Theorem 3.5 we have

$$
\delta\left(e_{0}^{d}\right)=\sum_{k=1}^{d-1}\left(h_{\frac{1}{d-k}} e_{\frac{-1}{k}}+e_{\frac{1}{d-k}} h_{\frac{-1}{k}}\right)+\sum_{k=1}^{d-2} x_{k} h_{1 / k} h_{0} h_{-1 /(d-k-1)}
$$

where $x_{1}, \ldots, x_{d-2} \in \mathbb{Z} / 2$ are unknown coefficients. The summands in the first sum on the right side all contribute to $\delta_{1}\left(e^{d}\right)$. To see this, we compute from equation (47) that

$$
\widetilde{\eta}\left(h_{\frac{1}{d-k}} e_{\frac{-1}{k}}\right)=-1 .
$$

On the other hand, by Lemma 4.8 and Proposition 3.2 .

$$
\begin{aligned}
I_{0}\left(e^{d}, h_{\frac{1}{d-k}} e_{\frac{n k-1}{k}}\right) & =I\left(e_{0}^{d-k} e_{n}^{k}, h_{\frac{1}{d-k}} e_{\frac{n k-1}{k}}\right) \\
& =1-2 d,
\end{aligned}
$$

where the right hand side denotes the index for the cylinder. Likewise for $e_{\frac{1}{d-k}} h_{\frac{n k-1}{k}}$. Thus

$$
\delta_{1}\left(e^{d}\right)=\sum_{k=1}^{d-1}\left(h_{\frac{1}{d-k}} e_{\frac{n k-1}{k}}+e_{\frac{1}{d-k}} h_{\frac{n k-1}{k}}\right)+h(\cdots) .
$$

Unless $n=1$ and $k=1, d-1$, we have

$$
E(\epsilon, n-\epsilon ; n k, d)=e_{\frac{1}{d-k}} e_{\frac{n k-1}{k}} .
$$

Then it follows by Theorem 3.1 that $h_{1 /(d-k)} e_{(n k-1) / k}$ and $e_{1 /(d-k)} h_{(n k-1) / k}$ are homologous in $C P_{*}(\epsilon, n-\epsilon ; n k, d)$. Hence, for all $n$ and $k, \delta_{1}\left(e^{d}\right)$ is $\delta_{0}$ homologous to a sum of generators each containing an $e$ or an $h$. So in terms of the filtration in the proof of Lemma 4.9] we have $\left(\delta_{1}\right)_{*}\left[e^{d}\right] \in H_{2 d-1}\left(\mathcal{F}_{d-1}\right)$, but by (49), $H_{2 d-1}\left(\mathcal{F}_{d-1}\right)=0$. 


\section{PFH of Dehn twists on higher genus surfaces}

Let $\Sigma$ be a compact connected symplectic surface, possibly with boundary. Choose a decomposition $\partial \Sigma=\partial_{+} \Sigma \sqcup \partial_{-} \Sigma$. Choose a finite number of disjoint embedded circles $\gamma_{i} \subset \Sigma$, and to each circle $\gamma_{i}$ associate a nonzero integer $n_{i}$. In this section we study the PFH in degree $d$ of the composition of $n_{i}$ positive Dehn twists along $\gamma_{i}$ for each $i$, for a perturbation which is a small positive rotation on $\partial_{+} \Sigma$ and a small negative rotation on $\partial_{-} \Sigma$.

\section{$5.1 \quad$ The setup}

To be more precise, we define $\phi^{\Sigma}: \Sigma \rightarrow \Sigma$ as follows.

First, let $N_{i}$ be disjoint tubular neighborhoods of the circles $\gamma_{i}$ with coordinates $x_{i} \in\left[-\epsilon,\left|n_{i}\right|+\epsilon\right]$ and $y_{i} \in \mathbb{R} / \mathbb{Z}$. Here $0<\epsilon<1 / d$. On the cylinder $N_{i}$, consider the twist

$$
\begin{aligned}
\phi_{i}^{0}: N_{i} & \longrightarrow N_{i}, \\
\left(x_{i}, y_{i}\right) \longmapsto & \longmapsto\left(x_{i}, y_{i}-x_{i}\right) .
\end{aligned}
$$

If $n_{i}>0$, let $\phi_{i}$ be a perturbation of $\phi_{i}^{0}$ as in Section 3.1 with $Q=d$. If $n_{i}<0$, let $\phi_{i}$ be the inverse of this perturbation.

Second, let $T_{i}:=\left(\epsilon,\left|n_{i}\right|-\epsilon\right) \times \mathbb{R} / \mathbb{Z} \subset N_{i}$, and let $\Sigma^{\prime}:=\Sigma \backslash \bigcup_{i} T_{i}$. Choose a Morse function $f: \Sigma^{\prime} \longrightarrow[0,1]$ such that

$$
\begin{aligned}
& f^{-1}(1)=\partial_{+} \Sigma \cup \bigcup_{n_{i}>0} \partial \overline{T_{i}}, \\
& f^{-1}(0)=\partial_{-} \Sigma \cup \bigcup_{n_{i}<0} \partial \overline{T_{i}},
\end{aligned}
$$

and $|\nabla f|=1$ near the boundary. Let $\phi_{f}: \Sigma^{\prime} \longrightarrow \Sigma^{\prime}$ be the time-1 Hamiltonian flow of $f$. Choose a Riemannian metric on $\Sigma^{\prime}$ which is large with respect to $d$. We assume that $f$ and the metric are Morse-Smale, and we let $\partial^{\text {Morse }}$ denote the mod 2 differential in the Morse complex.

We can make the above choices such that $\phi_{i}$ agrees with $\phi_{f}$ on $N_{i} \backslash T_{i}$, so the $\phi_{i}$ 's and $\phi_{f}$ patch together to give a symplectomorphism $\phi^{\Sigma}: \Sigma \rightarrow \Sigma$.

The period $\leq d$ periodic orbits of $\phi^{\Sigma}$ consist of fixed points at the critical points of $f$ in $\Sigma^{\prime}$, as well as, in each $T_{i}$, one elliptic and one hyperbolic orbit of period $q$ for each rational number $p / q \in\left(0,\left|n_{i}\right|\right)$ with $q \leq d$. 
We choose a generic almost complex structure $J$ on $Y_{\phi^{\Sigma}}$, such that $\left(\phi^{\Sigma}, J\right)$ is $d$ regular. For convenience, we assume that over $\Sigma^{\prime}$, the almost complex structure $J$ is close to the almost complex structure $J_{0}$ induced by the metric and $\omega$ via the identification $Y_{\phi_{f}} \simeq S^{1} \times \Sigma^{\prime}$ coming from the Hamiltonian isotopy. (One can drop this assumption by a modification of Section [5.2])

Lemma 5.1 Under the assumption (*) of Section [1] if $\alpha$ is a degree $d$ orbit set, then $\left(\phi^{\Sigma},[\alpha]\right)$ is monotone as in (8).

Proof We have a short exact sequence

$$
0 \longrightarrow H_{2}(\Sigma) \longrightarrow H_{2}\left(Y_{\phi^{\Sigma}}\right) \longrightarrow \operatorname{Ker}\left(1-H_{1}\left(\phi^{\Sigma}\right)\right) \longrightarrow 0 .
$$

Assumption $(*)$ implies that $\operatorname{Ker}\left(1-H_{1}\left(\phi^{\Sigma}\right)\right)$ is the image of the inclusioninduced map $H_{1}\left(\Sigma^{\prime}\right) \rightarrow H_{1}(\Sigma)$. The short exact sequence then has a splitting $\operatorname{Ker}\left(1-H_{1}\left(\phi^{\Sigma}\right)\right) \rightarrow H_{2}\left(Y_{\phi^{\Sigma}}\right)$, sending a loop $\xi \subset \Sigma^{\prime}$ to $S^{1} \times \xi \subset S^{1} \times \Sigma^{\prime} \simeq Y_{\phi^{f}}$. Since $\phi^{f}$ is Hamiltonian isotopic to the identity on $\Sigma^{\prime}$, $[\omega]$ vanishes on the image of this splitting, as does $c([\alpha])$. So we just have to check that if $\partial \Sigma=\emptyset$ then

$$
\int_{\Sigma} \omega=\lambda\langle[\Sigma], c([\alpha])\rangle \text {. }
$$

This holds if $d \neq g(\Sigma)-1$, because $\langle[\Sigma], c([\alpha])\rangle=2(d-g(\Sigma)+1)$.

Thus under assumption $(*)$, we have a well defined $\mathbb{Z} / 2(d-g(\Sigma)+1)$-graded chain complex

$$
\left(C P_{*}\left(\phi^{\Sigma}, d\right), \delta\right):=\bigoplus_{h \cdot[\Sigma]=d}\left(C P_{*}\left(\phi^{\Sigma}, h\right), \delta\right)
$$

whose differential $\delta$ may depend on $J$.

\subsection{The $\eta=(0, \ldots, 0)$ complex}

Continue to assume $(*)$. We now describe a differential $\delta_{0}$ on $C P_{*}\left(\phi^{\Sigma}, d\right)$, which is given explicitly in terms of Morse theory on $\Sigma^{\prime}$ and the cylinder complex for the $N_{i}$ 's, and which in some cases has the same homology as $\delta$.

Label the components of $\Sigma^{\prime}$ by $\left\{\Sigma_{j} \mid j=1, \ldots, \# \pi_{0} \Sigma^{\prime}\right\}$. For each $j$, let $\rho_{j} \subset Y_{\phi^{\Sigma}}$ be a circle obtained from $S^{1} \times\left\{z_{j}\right\} \subset S^{1} \times \Sigma_{j}$, where $z_{j}$ is not a fixed point of $\phi^{\Sigma}$. If $C$ is a flow line, define the wrapping number

$$
\eta_{j}(C):=\#\left(C \cap\left(\mathbb{R} \times \rho_{j}\right)\right) \in \mathbb{Z} .
$$


This does not depend on the choice of $z_{j}$. Define $\eta(C):=\left(\eta_{1}(C), \eta_{2}(C), \ldots\right)$.

We remark that if $\Sigma_{j}$ contains a component of $\partial \Sigma$, then any flow line $C$ automatically has $\eta_{j}(C)=0$, since one can choose $z_{j}$ near $\partial \Sigma$.

As in Lemma 4.3, we have $\eta_{j}(C) \geq 0$. Let $\delta_{0}$ denote the sum of the contributions to $\delta$ from flow lines $C$ with $\eta(C)=(0, \ldots, 0)$. Since $\eta_{j}(C) \geq 0$ and $\eta$ is additive under gluing of flow lines, $\delta^{2}=0$ implies $\delta_{0}^{2}=0$.

Lemma 5.2 Let $\alpha$ and $\beta$ be generators of $C P_{*}\left(\phi^{\Sigma}, d\right)$. Then $\left\langle\delta_{0} \alpha, \beta\right\rangle=1$ if and only if either:

(a) $\alpha=p \gamma$ and $\beta=q \gamma$, where $p, q \in \operatorname{Crit}(f)$ and $\left\langle\partial^{\text {Morse }} p, q\right\rangle=1$, or:

(b) $\alpha=\alpha^{\prime} \gamma$ and $\beta=\beta^{\prime} \gamma$, where $\alpha^{\prime}, \beta^{\prime}$ are products of orbits in some $N_{i}$, $\left\langle\delta \alpha^{\prime}, \beta^{\prime}\right\rangle=1$ in $C P_{*}\left(\phi_{i}\right)$, and $\gamma$ is a product of orbits outside $N_{i}$.

Proof We proceed in two steps.

Step 1 Let $C$ be an index one flow line with $\eta(C)=(0, \ldots, 0)$ and without trivial cylinders. We claim that $C$ is a flow line for $\phi_{f}$ or for some $\phi_{i}$.

There is a canonical trivialization $\tau$ of $V$ over the $N_{i}$ 's, and also over the circle corresponding to each critical point of $f$. There is no obstruction to extending this trivialization over $\Sigma \backslash\left\{z_{1}, z_{2}, \ldots\right\}$, so $\eta(C)=(0, \ldots, 0)$ implies that $c_{\tau}([C])=0$. It then follows as in (26) that

$$
2 g(C)+2 e_{-}(C)+h(C)=3 .
$$

Here $h(C)$ denotes the total number of ends of $C$ at hyperbolic orbits; and $e_{-}(C)$ denotes the number of incoming ends at elliptic orbits in $T_{i}$ 's with $n_{i}>0$ or at minima of $f$, plus the number of outgoing ends at elliptic orbits in $T_{i}$ 's with $n_{i}<0$ or at maxima of $f$.

If our claim fails, then since $C$ is connected, WLOG there exists $i$ with $n_{i}>0$ such that $C$ intersects $N_{i}$ in both the regions $\left\{x_{i}=-\epsilon / 2\right\}$ and $\left\{x_{i}=+\epsilon / 2\right\}$. As in Lemma 4.3. $C$ must have incoming hyperbolic and elliptic ends at $x_{i}=0$. Then $C$ cannot intersect the region $\left\{x_{i}=n_{i}-\epsilon / 2\right\}$, or else as in Lemma 4.3 again, $C$ would have an incoming end at $x_{i}=n_{i}$, violating (50). In the notation of equation (28), $[C \cap\{x=\epsilon / 2\}]=(0,1)$, so by equation (28), $C$ has an outgoing end inside $T_{i}$. Since this outgoing end is at $x_{i}>0$, equation (28) again implies that there is another incoming end inside $T_{i}$, contradicting (50). 
Step 2 We claim now that in $C P_{*}\left(\phi_{f}\right)$, if $\alpha$ and $\beta$ are generators then $\langle\delta \alpha, \beta\rangle=1$ if and only if (a) holds. This follows from standard arguments in Floer theory, cf. [12]. Namely, if $\gamma: \mathbb{R} \rightarrow \Sigma^{\prime}$ is a gradient flow line of $f$, then

$$
S^{1} \times \operatorname{graph}(\gamma) \subset S^{1} \times \mathbb{R} \times \Sigma^{\prime} \simeq \mathbb{R} \times Y_{\phi_{f}}
$$

is an embedded $J_{0}$-holomorphic cylinder cut out transversely, whose $\mathrm{PFH}$ index agrees with the Morse index. Index 1 flow lines not of this form may exist for $\left(\phi_{f}, J_{0}\right)$, but $S^{1}$ acts nontrivially on their moduli spaces, so by virtual cycle machinery as developed e.g. in [2, 7], the mod 2 count of such bad flow lines is zero after perturbing $J_{0}$ to our generic almost complex structure $J$.

\subsection{A single nonseparating Dehn twist on a closed surface}

Now let $\Sigma$ be a closed surface of genus $g$. We consider a single $(n=1)$ positive Dehn twist along a nonseparating circle $\gamma \subset \Sigma$, with neighborhoods $T \subset N$ and $\Sigma^{\prime}=\Sigma \backslash T$ as before.

Theorem 5.3 If $\phi^{\Sigma}$ is a nonseparating positive Dehn twist on a closed surface $\Sigma$ as above, and if $g \geq 2 d+1$, then as $\mathbb{Z} / 2(d-g+1)$-graded modules,

$$
H P_{*}\left(\phi^{\Sigma}, d\right) \simeq \Lambda^{d} K \oplus \bigoplus_{d^{\prime}=1}^{d} \Lambda^{d-d^{\prime}} K \otimes\left\{\mathbb{Z} / 2 \text { in index } 0, \ldots, 2 d^{\prime}-1\right\},
$$

where $K:=\operatorname{Ker}\left(H_{1}\left(\Sigma^{\prime}, \partial \Sigma^{\prime}\right) \rightarrow H_{0}\left(\partial \Sigma^{\prime}\right)\right) \otimes \mathbb{Z} / 2$.

Proof We proceed in 2 steps.

Step 1 We first show that $H_{*}\left(\delta_{0}\right)$ agrees with the right hand side of (51).

Let $e_{0}, e_{1}, h_{0}, h_{1}$ denote the elliptic and hyperbolic fixed points at $x=0,1$ in $N$. Our Morse function $f$ on $\Sigma^{\prime}$ will have minima at $e_{0}$ and $e_{1}$ and saddle points at $h_{0}$ and $h_{1}$. We choose $f$ to have no other minima, a unique maximum $m$ with $\partial^{\operatorname{Morse}}(m)=h_{0}+h_{1}$, one saddle point $s$ with $\partial^{\text {Morse }}(s)=e_{0}+e_{1}$, and $2(g-1)$ other saddle points in the kernel of $\partial^{\text {Morse }}$.

We define a filtration on the $\eta=0$ chain complex by setting $\mathcal{F}_{i}$ to be the span of those generators containing at least $d-i$ factors corresponding to critical points of $f$ in $\Sigma^{\prime}$. This gives rise to a spectral sequence with

$$
\mathcal{E}^{1} \simeq \bigoplus_{p=0}^{d} H P_{*}\left(\phi_{f}, d-p\right) \otimes H P_{*}\left(\left.\phi^{\Sigma}\right|_{T}, p\right)
$$


by Lemma 5.2. In the Morse complex of $f, e_{0}$ and $e_{1}$ represent the same homology class $e$, and $h_{0}$ and $h_{1}$ represent the same homology class $h$, so

$$
H P_{*}\left(\phi_{f}, d-p\right) \simeq \Lambda^{d-p} K \oplus \bigoplus_{l=1}^{d-p} \Lambda^{d-p-l} K \otimes \operatorname{span}\left\{e^{l}, e^{l-1} h\right\}
$$

by Lemma 5.2. It follows from the above two equations that

$$
\mathcal{E}^{1} \simeq \Lambda^{d} K \oplus \bigoplus_{d^{\prime}=1}^{d} \Lambda^{d-d^{\prime}} K \otimes \widehat{\mathcal{E}}^{1}\left(d^{\prime}\right),
$$

where $\widehat{\mathcal{E}}\left(d^{\prime}\right)$ denotes the $\eta=0$ spectral sequence for the torus from Lemma 4.9 with $n=1$, in degree $d^{\prime}$.

By Lemma 5.2, the higher differentials on $\mathcal{E}$ are determined by flow lines in $N$, and hence they are given by the differentials on $\widehat{\mathcal{E}}$, tensored with the identity on the $\Lambda^{*} K$ factors. Together with Lemma 4.9 this proves our claim.

Step 2 The theorem is trivial if $d=0$, so assume $d>0$. We now relate $H_{*}(\delta)$ to $H_{*}\left(\delta_{0}\right)$ using the wrapping spectral sequence from Lemma 4.4. If $C$ is a flow line from $\alpha$ to $\beta$, we can write

$$
I(C)=I_{0}(\alpha, \beta)+2(d-g+1) \eta(C) .
$$

Therefore $H_{*}\left(\delta_{0}\right)$ is $\mathbb{Z}$-graded, and if $\delta_{i}$ denotes the contribution to $\delta$ from flow lines $C$ with $\eta(C)=i$, then $\delta_{i}$ shifts the grading by $2(d-g+1) i-1$. Thus Lemma 4.4 is applicable as $d-g+1 \neq 0$. Since the $\eta=0$ homology is supported in index $0, \ldots, 2 d-1$, the wrapping spectral sequence will automatically degenerate at $\mathcal{E}^{1}$, giving $H_{*}(\delta) \simeq H_{*}\left(\delta_{0}\right)$, provided that

$$
|2(d-g+1) i-1|>2 d-1
$$

for all $i>0$, i.e. if $g \geq 2 d+1$.

\subsection{A single separating Dehn twist on a closed surface}

Now let $\Sigma$ be a closed surface, and consider a single $(n=1)$ positive Dehn twist along a separating circle $\gamma \subset \Sigma$. Departing slightly from the previous notation, denote the components of $\Sigma^{\prime}$ by $\Sigma_{0}$ and $\Sigma_{1}$, and let $g_{j}:=g\left(\Sigma_{j}\right)$.

Theorem 5.4 If $\phi^{\Sigma}$ is a separating positive Dehn twist on a surface as above, and if $g_{0}, g_{1} \geq 2 d$, then as $\mathbb{Z} / 2\left(d-g_{0}-g_{1}+1\right)$-graded modules,

$$
H P_{*}\left(\phi^{\Sigma}, d\right) \simeq \bigoplus_{q=0}^{d} \Lambda^{d-q} H_{1}\left(\Sigma^{\prime}\right) \otimes \bigoplus_{p=0}^{q} C_{p, q}\left[p^{2}-p\left(1-2 g_{0}+2 d\right)\right] .
$$


Here, if $p \leq q / 2$, then $C_{p, q}$ has one generator in each degree $0,2, \ldots, 2 p$, and also $2 q-1,2 q-3, \ldots, 2(q-p)+1$ if $p>1$; and $C_{p, q}=C_{q-p, q}$.

Proof We proceed in three steps.

Step 1 We first compute the $\eta=(0,0)$ homology.

Our Morse function $f$ on $\Sigma$ will have saddle points at $h_{0}$ and $h_{1}$ and minima at $e_{0}$ and $e_{1}$. We choose our labeling so that $e_{j}, h_{j} \in \Sigma_{j}$. We can assume that the only other critical points of $f$ are a maximum $m_{j} \in \Sigma_{j}$ with $\partial^{\text {Morse }} m_{j}=h_{j}$, and $2\left(g_{0}+g_{1}\right)$ saddle points with $\delta^{\text {Morse }}=0$.

Note that if there exists an $\eta=(0,0)$ flow line from $\alpha$ to $\beta$, then for homological reasons, $\alpha$ and $\beta$ have the same total numerator, where we regard critical points in $\Sigma_{j}$ as having numerator $j$. Let us redefine the saddle points to have numerator zero; then since the saddle points are in the kernel of $\delta^{\text {Morse }}$, it is still true by Lemma 5.2 that if $\left\langle\delta_{0} \alpha, \beta\right\rangle=1$ then $\alpha$ and $\beta$ have the same total numerator. Thus we have a well-defined, relatively $\mathbb{Z}$-graded subcomplex spanned by generators with total numerator $p$. We denote its homology by $H_{*}\left(\delta_{0}\right)(p)$, and we choose an absolute $\mathbb{Z}$-grading $I_{0}$ by declaring $I_{0}\left(e_{0}^{d-p} e_{1}^{p}\right)=0$. We claim then that, whether or not $g_{0}, g_{1} \geq 2 d$, we have

$$
H_{*}\left(\delta_{0}\right)(p) \simeq \bigoplus_{q=p}^{d} \Lambda^{d-q} H_{1}\left(\Sigma^{\prime}\right) \otimes C_{p, q}
$$

To prove (53), it is enough to do the case when $g_{0}=g_{1}=0$; the general case is obtained by including the $2\left(g_{0}+g_{1}\right)$ saddle points with $\partial^{\text {Morse }}=0$, which give rise to the $\Lambda^{*} H_{1}\left(\Sigma^{\prime}\right)$ factors.

As in Section [5.3, we compute $H_{*}\left(\delta_{0}\right)(p)$ using the spectral sequence $\mathcal{E}(p)$ coming from the filtration by the total degree in $\Sigma^{\prime}$. We have

$$
\mathcal{E}^{1}(p)=\bigoplus_{q=p}^{d} \bigoplus_{0 \leq r \leq p, d-q} H P_{*}(\epsilon, 1-\epsilon ; p-r, q) \otimes \operatorname{span}\left\{e_{0}^{d-q-r} e_{1}^{r}\right\}
$$

A somewhat lengthy calculation as in Lemma 4.9 then establishes (53).

Step 2 The theorem is trivial if $d=0$, so assume that $d>0$. We claim now that if $g_{0}, g_{1} \geq 2 d$, and if $Z \in H_{2}(Y ; \alpha, \beta)$ is a relative homology class with $\eta(Z)=\left(\eta_{0}, \eta_{1}\right) \neq(0,0)$ and $\eta_{i} \geq 0$, then

$$
I_{0}(\alpha)-I_{0}(\beta) \geq I(\alpha, \beta ; Z)+2 d-1 .
$$


To prove the claim, WLOG we can take $\alpha=e_{0}^{d-p} e_{1}^{p}$ and $\beta=e_{0}^{d-p-k} e_{1}^{p+k}$, with $0 \leq k \leq d-p \leq d$. We must show that $I(\alpha, \beta ; Z) \leq-2 d+1$. For the trivialization $\tau$ in Section 5.2 we have

$$
c_{\tau}(Z)=\eta_{0}\left(1-2 g_{0}\right)+\eta_{1}\left(1-2 g_{1}\right) .
$$

A calculation as in equation (24) shows that

$$
Q_{\tau}(Z)=k(d-2 p-k)+d\left(\eta_{0}+\eta_{1}\right) .
$$

Of course, $\mu_{\tau}(\alpha, \beta)=0$. For homological reasons, we have $\eta_{0}-\eta_{1}=k$. Putting this all together, we get

$$
I(\alpha, \beta ; Z)=k\left(1-2 g_{0}+2 d-2 p-k\right)+\eta_{1}\left(2-2 g_{0}-2 g_{1}+2 d\right) .
$$

If $k=0$, then $\eta_{1}>0$, so $g_{0}, g_{1} \geq 2 d$ implies that $I(\alpha, \beta ; Z) \leq-6 d+2$. If $k \geq 1$, then using $g_{0} \geq 2 d, p \geq 0, \eta_{1} \geq 0$, and $g_{1} \geq 2 d>0$, we get $I(\alpha, \beta ; Z) \leq-2 d$. Either way, our claim holds.

Step 3 By Step 2, we can write $\delta=\delta_{0}+\delta_{1}+\cdots$ where $\delta_{i}$ shifts the $\mathbb{Z}$ grading $I_{0}$ on the $\eta=(0,0)$ complex by $-2 i-1$, and $\delta_{1}=\cdots=\delta_{d-1}=0$. It follows from Lemma 4.4 that the wrapping spectral sequence is well defined with $N=-2$; and it automatically degenerates at $\mathcal{E}^{1}$, since the $\eta=(0,0)$ homology is supported in degree $0, \ldots, 2 d-1$ by Step 1 . Thus, by (53), we have

$$
H P_{*}\left(\phi^{\Sigma}, d\right) \simeq \bigoplus_{q=0}^{d} \Lambda^{d-q} H_{1}\left(\Sigma^{\prime}\right) \otimes \bigoplus_{p=0}^{q} C_{p, q}
$$

as $\mathbb{Z} / 2$-graded modules. Now every differential in the wrapping spectral sequence has degree -1 with respect to the $\mathbb{Z} / 2\left(d-g_{0}-g_{1}+1\right)$-grading. By (54), we can refine (55) to an isomorphism of $\mathbb{Z} / 2\left(d-g_{0}-g_{1}+1\right)$-graded modules by inserting grading shifts as in (52).

\subsection{The general case}

For a composition of several Dehn twists along disjoint circles, as considered in Section [5.1, arguments like the above show that the wrapping spectral sequence will exist and automatically degenerate at $\mathcal{E}^{1}$, provided that condition (**) from Section 1 holds. Then in principle the homology can be computed combinatorially using Lemma 5.2 as illustrated in the specific cases above.

Without condition $(* *)$, one would need to understand the contribution from flow lines with $\eta \neq(0, \ldots, 0)$. For all we know these might never contribute 
to the differential, except when some $\Sigma_{j}$ has genus 0 and does not contain a component of $\partial \Sigma$.

Without condition $(*)$, monotonicity often fails. (Our composition of Dehn twists will still have a monotone representative of its symplectic isotopy class for each homology class $h$ as long as $\partial \Sigma \neq \emptyset$ or $d \neq g-1$; however there might be no such representative which is in standard form, i.e. equal to the identity away from the twisting circles.) Without monotonicity one can still define a version of $\mathrm{PFH}$ with coefficients in an appropriate Novikov ring over $\mathrm{H}_{2}(Y)$, which can be computed using the above methods.

It is an interesting problem to attempt to extend these results to compositions of Dehn twists along intersecting circles. It would also be interesting to try to compute $\mathrm{PFH}$ of pseudo-Anosov maps in terms of hyperbolic geometry.

\section{A An argument from Morse-Bott theory}

Recall from (18) that we can identify

$$
\mathbb{R} \times Y_{\phi_{0}} \simeq \mathbb{R} \times S^{1} \times\left[X_{1}, X_{2}\right] \times S^{1} .
$$

We denote the coordinates on the right hand side by $s, t, x, y$. There is a natural almost complex structure $J_{0}$ on the right hand side defined by

$$
\begin{aligned}
& J_{0}\left(\partial_{s}\right):=R, \\
& J_{0}\left(\partial_{x}\right):=\partial_{y} .
\end{aligned}
$$

Here $R=\partial_{t}-x \partial_{y}$ denotes the mapping torus flow. Also $J_{0}$ is invariant under the map $(s, t, x, y) \mapsto(s, t, x+n, y-n t)$, and hence descends to $\mathbb{R} \times Y_{\phi_{0}^{T}}$.

We can perform a perturbation of $\left(\phi_{0}, J_{0}\right)$ or $\left(\phi_{0}^{T}, J_{0}\right)$ to obtain a pair $(\phi, J)$ such that $\phi$ satisfies the conditions in Section 3.1. and $J$ is admissible (but $(\phi, J)$ is not necessarily locally linear). In this appendix, we use Morse-Bott theory for the unperturbed setup to prove the following lemma. This explains Remark 3.6 and is used in the proof of Lemma 4.5

\section{Lemma A.1}

(a) There is a generic perturbation $(\phi, J)$ of $\left(\phi_{0}, J_{0}\right)$ as above such that if $\alpha$ and $\beta$ are generators of $C P_{*}\left(X_{1}, X_{2} ; P, Q\right)$, and if $\alpha$ is obtained from $\beta$ by double rounding, then genus 0 flow lines in $\mathcal{M}(\alpha, \beta)$ do not exist. 
(b) For every positive integer $q$, there is a generic perturbation $(\phi, J)$ of $\left(\phi_{0}^{T}, J_{0}\right)$ as above, for which there do not exist any genus 1 flow lines with all outgoing ends elliptic and with a single incoming end at a hyperbolic orbit $h_{p / q}$ of multiplicity one.

Proof (a) For the unperturbed map $\phi_{0}$, let $\gamma_{p / q}$ denote the circle of periodic orbits at $x=p / q$. It will be convenient for Lemma A.2 below to define an explicit diffeomorphism $\theta: \gamma_{p / q} \rightarrow S^{1}$ by sending an orbit $\gamma \in \gamma_{p / q}$ to

$$
\theta(\gamma):=q y+p t+\frac{p q}{2} \in \mathbb{R} / \mathbb{Z}
$$

for any point $(t, x=p / q, y) \in \gamma$. This is well-defined since $y+x t$ is constant on $\gamma$.

Suppose we are given rational numbers $p_{1} / q_{1}, \ldots, p_{k} / q_{k}$ (not necessarily distinct), positive integers $\lambda_{1}, \ldots, \lambda_{k}$, and also $p_{1}^{\prime} / q_{1}^{\prime}, \ldots, p_{l}^{\prime} / q_{l}^{\prime}$ and $\lambda_{1}^{\prime}, \ldots, \lambda_{l}^{\prime}$. Let $\widetilde{\mathcal{M}}$ denote the moduli space of generalized flow lines for the unperturbed setup with ordered ends, consisting of an outgoing end of multiplicity $\lambda_{i}$ at some periodic orbit in the circle $\gamma_{p_{i} / q_{i}}$, an incoming end of multiplicity $\lambda_{j}^{\prime}$ at some periodic orbit in the circle $\gamma_{p_{j}^{\prime} / q_{j}^{\prime}}$, and no other ends. There is an "endpoint map"

$$
\xi: \widetilde{\mathcal{M}} / \mathbb{R} \longrightarrow \prod_{i=1}^{k} \gamma_{p_{i} / q_{i}} \times \prod_{j=1}^{l} \gamma_{p_{j}^{\prime} / q_{j}^{\prime}}
$$

which sends a flow line to the periodic orbits at its ends. Given $C \in \widetilde{\mathcal{M}}$, write

$$
\theta \circ \xi(C)=\left(\theta_{1}, \ldots, \theta_{k}, \theta_{1}^{\prime}, \ldots, \theta_{l}^{\prime}\right) \in\left(S^{1}\right)^{k+l} .
$$

Lemma A.2 The endpoints of any $C \in \widetilde{\mathcal{M}}$ satisfy the linear relation

$$
\sum_{i=1}^{k} \lambda_{i} \theta_{i}=\sum_{j=1}^{l} \lambda_{j}^{\prime} \theta_{j}^{\prime} \in \mathbb{R} / \mathbb{Z} .
$$

Proof It follows from (56) that the 2 -form $d t d y-d s d x$ on $\mathbb{R} \times Y_{\phi_{0}}$ annihilates any pair of tangent vectors of the form $\left(v, J_{0} v\right)$. Therefore

$$
\int_{C} d t d y=\int_{C} d s d x
$$

Now $\int_{C} d s d x=0$ by Stokes theorem, because the 1-form $s d x$ vanishes along the periodic orbits. Therefore $\int_{C} d t d y=0$. But $\int_{C} d t d y$ is just the area of the 
projection of $C$ to the $(t, y)$-torus, and so under the identification (57),

$$
\int_{C} d t d y \equiv \sum_{i=1}^{k} \lambda_{i} \theta_{i}-\sum_{j=1}^{l} \lambda_{j}^{\prime} \theta_{j}^{\prime} \quad \bmod \mathbb{Z} .
$$

The reason is that modulo $\mathbb{Z}$, the area of a homology in the $(t, y)$-torus between a periodic orbit and a linear combination of the curves $y=0$ and $t=0$ is equal to the right hand side of (57).

We now relate the perturbed setup to the unperturbed setup. As in [1, we can perturb by choosing a Morse function $f_{p / q}$ on $\gamma_{p / q}$ with one index 1 critical point at $\tilde{h}_{p / q}$ and one index 0 critical point at $\tilde{e}_{p / q}$. Then $\tilde{e}_{p / q}$ and $\tilde{h}_{p / q}$ get perturbed into $e_{p / q}$ and $h_{p / q}$. Under the identification (57), it will be convenient below to choose $\theta\left(\tilde{e}_{p / q}\right)=0$ for all $p, q$, and set $\theta\left(\tilde{h}_{p / q}\right)$ equal to some fixed irrational number $\zeta$ between $2 / 3$ and 1 .

Suppose $\left(\phi_{n}, J_{n}\right)$ is a sequence of generic such perturbations converging to the unperturbed setup, for which genus 0 flow lines $C_{n}$ exist from $\alpha$ to $\beta$. We can pass to a subsequence such that all the $C_{n}$ 's have the same partitions at the ends. Then a compactness argument as in [1] shows that there exist GFL's $C_{0}^{\prime}, \ldots, C_{k}^{\prime}$ for the unperturbed setup such that:

(i) There is a bijection between the outgoing ends of $C_{0}^{\prime}$ and the outgoing ends of each $C_{n}$. If an outgoing end of each $C_{n}$ is at $e_{p / q}$, then the corresponding end of $C_{0}^{\prime}$ is at $\tilde{e}_{p / q}$.

(ii) For $0 \leq i<k$, there is a bijection between the incoming ends of $C_{i}^{\prime}$ and the outgoing ends of $C_{i+1}^{\prime}$. For each such pair, both such ends are on the same $\gamma_{p / q}$. Moreover, there is a downward flow line of $f_{p / q}$ from the incoming end to the outgoing end.

(iii) There is a bijection between the incoming ends of $C_{k}^{\prime}$ and the incoming ends of each $C_{n}$. If an incoming end of each $C_{n}$ is at $h_{p / q}$, then the corresponding end of $C_{k}^{\prime}$ is at $\tilde{h}_{p / q}$.

(iv) The surface obtained by gluing the $C_{i}^{\prime}$ 's together along their paired ends from (ii) has the same topological type as the $C_{n}$ 's.

As in Proposition 3.12 the polygonal path determined by the outgoing ends of a GFL cannot cross to the right of the polygonal path determined by the incoming ends. This allows us to classify the possibilities for the curves $C_{0}^{\prime}, \ldots, C_{k}^{\prime}$ as follows. Without loss of generality, $\beta=h_{p_{1}^{\prime} / q_{1}^{\prime}} h_{p_{2}^{\prime} / q_{2}^{\prime}} h_{p_{3}^{\prime} / q_{3}^{\prime}}$, and $\alpha$ is a product of $e_{p / q}$ 's. Furthermore the curves $C_{0}^{\prime}, \ldots, C_{k}^{\prime}$ contain a total of at most two 
components that are not trivial cylinders or branched covers thereof. There cannot be just one such component, because then that component would have all outgoing ends at $\tilde{e}_{p / q}$ 's and all incoming ends at $\tilde{h}_{p / q}$ 's, which contradicts Lemma A.2 since $\zeta$ is irrational. So there are two such components, call them $C_{+}$and $C_{-}$. We can order the factors in $\beta$ so that $C_{+}$has outgoing ends at some $\tilde{e}_{p / q}$ 's and incoming ends at $\tilde{h}_{p_{1}^{\prime} / q_{1}^{\prime}}$ and some orbit $x_{p_{0} / q_{0}} \in \gamma_{p_{0} / q_{0}}$; while $C_{-}$has outgoing ends at some $\tilde{e}_{p / q}$ 's and some orbit $y_{p_{0} / q_{0}} \in \gamma_{p_{0} / q_{0}}$, and incoming ends at $\tilde{h}_{p_{2}^{\prime} / q_{2}^{\prime}}$ and $\tilde{h}_{p_{3}^{\prime} / q_{3}^{\prime}}$. By Lemma $\$$.2

$$
\begin{aligned}
& \theta\left(x_{p_{0} / q_{0}}\right)=-\zeta \in(0,1 / 3), \\
& \theta\left(y_{p_{0} / q_{0}}\right)=2 \zeta \in(1 / 3, \zeta) .
\end{aligned}
$$

But by condition (ii) above, there is a downward flow line of $f_{p_{0} / q_{0}}$ from $\theta\left(x_{p_{0} / q_{0}}\right)$ to $\theta\left(y_{p_{0} / q_{0}}\right)$. This contradicts (58), since $f_{p_{0} / q_{0}}$ takes its maximum at $\zeta$ and its minimum at 0 .

(b) For $\phi_{0}^{T}$, the map (57) is not quite well-defined, but it does give a welldefined two-to-one map $\gamma_{p / q} \rightarrow \mathbb{R} / \frac{1}{2} \mathbb{Z}$. Then Lemma A.2 still holds in $\mathbb{R} / \frac{1}{2} \mathbb{Z}$. In particular, if we choose $\theta\left(\tilde{e}_{p / q}\right)=0$ and set $\theta\left(\tilde{h}_{p / q}\right)$ equal to some fixed irrational number, then there do not exist any GFL's for $\left(\phi_{0}^{T}, J_{0}\right)$ with all outgoing ends at $\tilde{e}_{p / q}$ 's and all incoming ends at $\tilde{h}_{p / q}$ 's.

Suppose $\left(\phi_{n}, J_{n}\right)$ is a sequence of generic perturbations as above converging to the unperturbed setup, for which genus 1 flow lines $C_{n}$ exist with a single incoming end at a hyperbolic orbit $h_{p / q}$ of multiplicity one, and all outgoing ends ellipitic. Since $q$ is assumed fixed, there are only finitely many possibilities for the ends of $C_{n}$, so we can pass to a subsequence such that the $C_{n}$ 's have outgoing ends at the same elliptic orbits with the same multiplicities, and an incoming end at the same hyperbolic orbit $h_{p_{-} / q_{-}}$. Then as before, there exist GFL's $C_{0}^{\prime}, \ldots, C_{k}^{\prime}$ for the unperturbed setup satisfying conditions (i)-(iv) above.

Some component of some $C_{i}^{\prime}$ must have genus 1 . Otherwise all the $C_{i}^{\prime}$ 's lift to the cylinder. As in Proposition 3.12, if a GFL for the cylinder has one incoming end, then the outgoing ends have the same underlying polygonal path. So by condition (iii) and downward induction on $i$, each $C_{i}^{\prime}$ is a trivial cylinder on $\tilde{h}_{p_{-} / q_{-}}$. This leads to multiple contradictions.

Since the $C_{n}$ 's also have genus 1 , it follows from condition (iv) that each component of each $C_{i}^{\prime}$ has only one incoming end. The genus zero components are then branched covers of trivial cylinders as above. This means that the genus 1 component has an incoming end at $\tilde{h}_{p_{-} / q_{-}}$and all outgoing ends at $\tilde{e}_{p / q}$ 's. This contradicts our choices of $\theta\left(\tilde{e}_{p / q}\right)$ and $\theta\left(\tilde{h}_{p / q}\right)$. 


\section{References}

[1] F. Bourgeois, A Morse-Bott approach to contact homology, Symplectic and contact topology: interactions and perspectives (Toronto, ON/Montreal, QC, 2001), 55-77, Fields Inst. Commun., 35, Amer. Math. Soc., Providence, RI, 2003. MathReview

[2] K. Cieliebak, I. Mundet i Riera, and D. A. Salamon, Equivariant moduli problems, branched manifolds, and the Euler class, Topology 42 (2003), 641-700. MathReview

[3] S. K. Donaldson, Floer homology and algebraic geometry, Vector bundles in algebraic geometry (Durham, 1993), 119-138, London Math. Soc. Lecture Note Ser., 208, Cambridge Univ Press, 1995. MathReview

[4] E. Eftekhary, Floer cohomology of certain pseudo-Anosov maps on surfaces. arXiv:math.SG/0205029

[5] Ya. Eliashberg, A. Givental, and H. Hofer, Ya. Eliashberg, A. Givental, and H. Hofer, Introduction to symplectic field theory, Geom. Funct. Anal. 2000, Special Volume, Part II, 560-673. MathReview

[6] A. Floer, Morse theory for Lagrangian intersections, J. Differential Geom. 28 (1988), no. 3, 513-547. MathReview

[7] K. Fukaya and K. Ono, Arnold conjecture and Gromov-Witten invariant, Topology 38 (1999), no. 5, 933-1048. MathReview

[8] R. Gautschi, Floer homology of algebraically finite mapping classes, J. Symplectic Geom. 1 (2003), 715-765. MathReview

[9] M. Hutchings, An index inequality for embedded pseudoholomorphic curves in symplectizations, J. Eur. Math. Soc. 4 (2002), 313-361. MathReview

[10] M. Hutchings and Y-J. Lee, Circle-valued Morse theory, Reidemeister torsion, and Seiberg-Witten invariants of 3-manifolds, Topology 38 (1999), no. 4, 861888. MathReview

[11] M. Hutchings and M. Sullivan, Rounding corners of polygons and the embedded contact homology of $T^{3}$. arXiv:math.SG/0410061

[12] M. Hutchings and M. Thaddeus, Periodic Floer homology, in preparation.

[13] S. Jabuka and T. Mark, Heegard Floer homology of certain mapping tori, Algebraic and Geometric Topology 4 (2004), 685-719. MathReview

[14] Y-J. Lee, Reidemeister torsion in symplectic Floer theory and counting pseudoholomorphic tori. arXiv:math.DG/0111313

[15] D. McDuff, Singularities and positivity of intersections of J-holomorphic curves, pp. 191-216 in Holomorphic curves in symplectic geometry (M. Audin and F. Lafontaine, ed.), Progress in Mathematics 117, Birkhäuser, 1994. MathReview

[16] P. Ozsváth and Z. Szabó, Holomorphic disks and topological invariants for closed 3-manifolds, Annals of Math. 159 (2004), 1027-1158. MathReview 
[17] P. Ozsváth and Z. Szabó, Holomorphic disks and three-manifold invariants: properties and applications, Annals of Math. 159 (2004), 1159-1245. MathReview

[18] P. Ozsváth and Z. Szabó, Holomorphic disks and knot invariants, Adv. Math. 186 (2004), 58-116. MathReview

[19] D. Salamon, Seiberg-Witten invariants of mapping tori, symplectic fixed points, and Lefschetz numbers, Proceedings of the 6th Gökova Geometry-Topology Conference. Turkish J. Math. 23 (1999), no. 1, 117-143. MathReview

[20] M. Schwarz. Cohomology operations from $S^{1}$ cobordisms in Floer homology, ETH Zürich thesis, 1995.

[21] P. Seidel, The symplectic Floer homology of a Dehn twist, Math. Res. Lett. 3 (1996), no. 6, 829-834. MathReview

[22] P. Seidel, More about vanishing cycles and mutation, Symplectic geometry and mirror symmetry (Seoul, 2000), 429-465, World Sci. Publishing. MathReview

[23] M. Sullivan, K-theoretic invariants for Lagrangian Floer homology, Geom. Funct. Anal. 12 (2002), 810-872. MathReview

[24] C. H. Taubes, The Seiberg-Witten and Gromov invariants, Math. Res. Lett. 2 (1995), no. 2, 221-238. MathReview

[25] C. H. Taubes, A compendium of pseudoholomorphic beasts in $\mathbb{R} \times\left(S^{1} \times S^{2}\right)$, Geometry and Topology 6 (2002), 657-814. MathReview

[26] C. H. Taubes, Pseudoholomorphic punctured spheres in $\mathbb{R} \times\left(S^{1} \times S^{2}\right)$ : properties and existence, preprint, 2004.

Department of Mathematics, University of California

Berkeley, CA 94720-3840, USA

and

Department of Mathematics and Statistics, University of Massachusetts

Amherst, MA 01003-9305, USA

Email: hutching@math.berkeley.edu and sullivan@math.umass.edu

Received: 9 October 2004 